Article

\title{
Linear or Nonlinear Modeling for ENSO Dynamics?
}

\author{
Marco Bianucci ${ }^{1, *} \mathbb{0}$, Antonietta Capotondi ${ }^{2}$, Riccardo Mannella ${ }^{3}$ and Silvia Merlino ${ }^{1}$ \\ 1 ISMAR-CNR, 19032 La Spezia, Italy; silvia.merlino@sp.ismar.cnr.it \\ 2 NOAA-ESRL, Physical Sciences Division, University of Colorado, CIRES, Boulder, CO 80305, USA; \\ antonietta.capotondi@noaa.gov \\ 3 Physics Department, Pisa University, 56127 Pisa, Italy; riccardo.mannella@unipi.it \\ * Correspondence: marco.bianucci@cnr.it
}

Received: 12 September 2018; Accepted: 4 November 2018; Published: 8 November 2018

\begin{abstract}
The observed ENSO statistics exhibits a non-Gaussian behavior, which is indicative of the presence of nonlinear processes. In this paper, we use the Recharge Oscillator Model (ROM), a largely used Low-Order Model (LOM) of ENSO, as well as methodologies borrowed from the field of statistical mechanics to identify which aspects of the system may give rise to nonlinearities that are consistent with the observed ENSO statistics. In particular, we are interested in understanding whether the nonlinearities reside in the system dynamics or in the fast atmospheric forcing. Our results indicate that one important dynamical nonlinearity often introduced in the ROM cannot justify a non-Gaussian system behavior, while the nonlinearity in the atmospheric forcing can instead produce a statistics similar to the observed. The implications of the non-Gaussian character of ENSO statistics for the frequency of extreme El Niño events is then examined.
\end{abstract}

Keywords: non-Hamiltonian complex systems; Fokker-Planck equation; transport coefficients; El Niño-La Niña; Lie evolution; projection approach

\section{Non-Gaussian-Nonlinear Features of the ENSO Statistics}

It is well established that some aspects of the El Niño Southern Oscillation (ENSO) have a non-Gaussian statistics, a characteristic that is indicative of some underlying nonlinear process. For example, the plot of the frequency of the Niño 3 index (averaged Sea Surface Temperature (SST) anomalies in the region $5^{\circ} \mathrm{S}-5^{\circ} \mathrm{N}, 150^{\circ} \mathrm{W}-90^{\circ} \mathrm{W}$ ) shows that the likelihood of large positive SST anomalies is greater than that of negative anomalies (Figure 1), indicating that the SST anomalies achieved in the eastern equatorial Pacific during the mature phase of an El Niño are larger than those occurring during la Niña events. Another asymmetry is the duration of the transition from one ENSO phase to the other, with a rapid transition from El Niño to La Niña, but a slower transition from La Niña to El Niño [1]. The duration of El Niño events tends to be shorter than that of La Niña ones, and the range of El Niño flavors is larger than that of La Niña [2].

This non-Gaussian behavior is quantified by the positive skewness of the SST distribution: $\gamma_{1} \sim 0.7$. A positive kurtosis $\gamma_{2} \sim 1.1$ also clearly indicates that extreme events are more likely to occur than for a normal distribution.

The non-Gaussianity of the histogram arises from non-linear contributions in the equation of motion governing the system, and it is important to understand which nonlinearities play the most relevant role in the ENSO evolution. We know that ENSO is the result of complex large-scale ocean-atmosphere interactions that also include non-linear processes. For instance, An and Jin [3] proposed that the Nonlinear Dynamical Heating (NDH) in the ocean mixed layer is crucial for generating intense El Niño events, mainly due to the vertical nonlinear advection. Asymmetries in intensity can also result from ocean-atmosphere coupling processes (e.g., [4]) or from the nonlinear 
response of the zonal wind stress to ENSO SST anomalies [5,6]. In general, the complexity of the ENSO, as for most of the large-scale phenomena resulting from the ocean-atmosphere interaction, makes it challenging to understand the general key features of the statistics and dynamics of the events. Thus, to mimic the highly complex nature of ENSO events, as the diversity in spatial flavors and the "non-normal" growth of fluctuations [7], large multivariate linear models with multivariate additive stochastic perturbations have been exploited $[8,9]$. Here, we focus instead on Low Order Models (LOMs), i.e., the set of Ordinary Differential Equations (ODE) for ENSO, perturbed by a fast and chaotic forcing. Probably, a more realistic modeling of ENSO should merge these two pictures (multidimensionality and nonlinearities). A deeper discussion about the effective and respective roles of the dimension and of the nonlinearities of an LOM to model the complexity of ENSO goes beyond the task of the present work.

The tropical Pacific can be well described by shallow water equations driven at the surface by the easterly trade winds, thus allowing for a drastic simplification of the system dynamics.

Simple shallow water equations together with the assumption of a large timescale separation between the ENSO dynamics and the typical dynamics of atmospheric processes make it possible to reduce the description of the dynamics of the ENSO-relevant variables, to a relatively simple LOM. This LOM can be separated in a slow part, or "system of interest", associated with the slow evolution of oceanic variables like SST and thermocline depth, and a fast "rest of the system" that primarily includes fast atmospheric processes, e.g., the Madden-Julian Oscillation (MJO), the Westerly Wind Bursts (WWBs), etc., which are considered important in forcing ENSO. ENSO LOMs that have been extensively considered in the literature include the Recharge Oscillator Model (ROM, e.g., [10-17] among many others) and the Delayed Oscillator (DO, e.g., $[18,19])$. In these models, ENSO events are triggered by the forcing provided by the fast atmospheric variables, which are usually represented as a stochastic perturbation of the system of interest.

The simplified representation of ENSO in the LOMs provides a suitable framework for examining the impact of the system nonlinearities on the ENSO statistics. In particular, an important question is whether the relevant nonlinearities reside in the interaction among the variables of the system of interest (dynamical nonlinearities), as suggested, for example in [18], or are associated with the interaction of the system of interest with the atmospheric fast forcing, as indicated, among others, by $[6,20]$.

The fast atmospheric forcing can be included in two ways: considering this forcing as stochastic or assuming a deterministic motion with initial conditions distributed according to some distribution (the Gibbs idea of ensemble). The former approach is often used in the oceanographic field, where the dynamics is divided into a slow and a very fast and chaotic component, and the fast part is considered to be random (usually modeled as white noise) [21-25]. The latter approach has been largely used in the context of the foundation of statistical mechanics and thermodynamics and, more recently, also in the oceanographic field by some of the authors $[17,26,27]$. It aims at starting from more realistic deterministic systems (instead of the stochastic ones), and by using projection- perturbation procedures, it allows managing also the cases in which the time scale separation between the dynamics of the part of interest and that of the rest of the system is not so large: this is the reason why in this work, we shall focus on the projection-perturbation approach, which has been applied to the multi-scale ENSO system.

Both approaches have their strengths and weaknesses, and a large literature has been devoted to justify both from a formal and theoretical point of view (for a review about the foundation of the stochastic approach, see [28], and for the projection approach, see, e.g., [29-33]). It is far beyond the scope of the present work to discuss the foundation of these approaches. However, both aim at obtaining a Partial Differential Equation (PDE), ideally a Fokker-Planck Equation (FPE), guiding the dynamics of the Probability Density Function (PDF) for the variables of the part of interest of the system. 
The FPE has been historically used to describe the PDF of Brownian-like particles [34,35]. Actually, the FPE has been recognized as a very useful tool for modeling a wide variety of stochastic phenomena arising in physics, chemistry, biology, finance, traffic flow, etc. [36]. The importance of the FPE cannot be overestimated, and in the classical books of stochastic methods, it is obtained from a truncating Kramers-Moyal expansion of the Markovian master equation [37-39]. From an FPE, it is possible to obtain statistical information about the quantity of interest. For example, the FPE is the major approach for determining, both analytically and numerically, important quantities like the First Passage Time (FPT; see for example [40]), in a variety of contexts ranging from civil and mechanical engineering, physical chemistry [41-43], disordered systems [44,45], neuroscience [46,47], biochemistry [48], biomedicine, mathematical financing, image processing, computer science, etc.

More generally, the possibility of using an FPE to model the statistical behavior of the relevant properties of the variables of interest is often crucial because the FPE is the only non-trivial partial differential equation that in many cases can be approached for a solution with analytical tools, and it looks like a Schrodinger equation [49]. In the present paper, we shall obtain a general FPE for the perturbed linear/non-linear ROM representing the ENSO dynamics, and we will compare some general statistical features resulting from the FPE (such as the stationary PDF, the relaxation properties and the average timing of the events) with observations, to infer the specific nature of the nonlinearities of the ENSO modeled by the ROM.

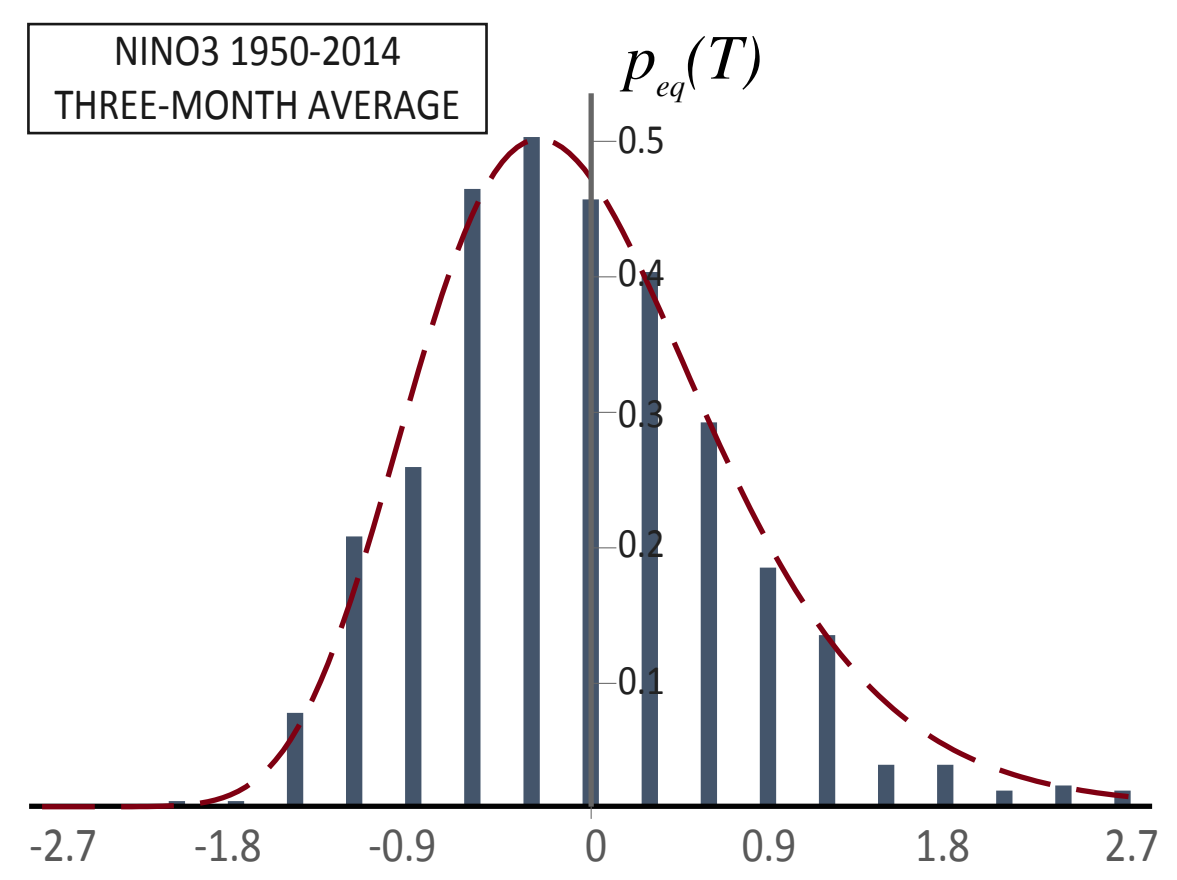

Figure 1. Histogram of the frequency for the three-month average Niño 3 data from NOAA [50]. The dashed line is the best fit of the stationary PDF found in [17] (strongly skewed and with a heavy tail), and solid blue line is a normal distribution with the same average and variance of the observation data.

The contents are organized as follows: Section 2 is devoted to reviewing and discussing the way the ROM emerges as an LOM describing the dynamics of the anomalies of the thermocline depth and of the SST of the equatorial Pacific Ocean. This section shows from both a physical and observational point of view that the ROM can provide a good representation of the ENSO dynamics. In Section 3, we start discussing the nonlinearities, and we present a first result of the present paper: comparing observations with the model, it is hard to justify/detect possibly internal dynamical nonlinearities for the ROM (nonlinear coupling between the ROM variables). In Section 4, we introduce and justify the nonlinear coupling between the unperturbed ROM and the fast (but not extremely fast) forcing (mostly 
by the atmosphere). In Section 5, starting from the perturbed ROM, we exploit recent results $[17,26,27]$ to obtain the explicit expression of the FPE for the ROM. This is mainly a review of previous results, but it is developed rather extensively here to make the present work self-contained. In Section 6, we use different methods (averages of first moments, covariance matrix, first passage time, power spectrum and expectation of the process time increments) to infer, from data, the values of the FPE coefficients, comparing them with the theoretical ones. The aim is to assess where the nonlinearities responsible for the non-Gaussian features of the ENSO statistics reside. Section 7 is devoted to discussing the results and drawing conclusions.

\section{The Dynamics of the Components of the ENSO: The Recharge Oscillator Model}

The El Niño Southern Oscillation, also known as ENSO, is a periodic fluctuation of the SST (El Niño) and the sea level pressure (Southern Oscillation) across the equatorial Pacific Ocean. The fluctuations arise over a normal condition of strong SST gradient between the east (cold tongue) and the west (warm pool) equatorial Pacific Ocean. ENSO is a complicated, not yet fully-understood, coupled ocean-atmosphere phenomenon in which basin-wide changes in sea surface temperatures, trade winds and atmospheric convection are intimately related. However, the particular ocean and atmosphere conditions that generate, and in some way also define, the El Niño/La Niña events allow the representation of the key ocean-atmospheric feedbacks responsible for this complex phenomenon in a simplified fashion.

The upper layer of the tropical Pacific Ocean, responsible for the energy and momentum exchange with the atmosphere, is a very long strip of water with a meridional width of a few hundred kilometers, a zonal extension of about 10,000 km and an average depth of about $120 \mathrm{~m}$, as defined by the depth of the thermocline. The water in this strip flows over the deeper and colder ocean, from the South American coasts to the warm Asian coasts, mainly driven by the easterly trade winds and "trapped" in the convergence ocean zone, around the equatorial latitude, ultimately due to the Coriolis effect.

This motion accumulates warm water in the western Pacific Ocean, and because of the closed eastern boundary, it forces the cold and deep water to rise up in the eastern Pacific to compensate the surface water moving westward. This process generates the strong SST gradient between the two sides of the basin, which, in normal conditions, is in equilibrium with the easterly trade winds.

During an El Niño (La Niña) event, this SST gradient from east to west is reduced (increased): we consider that an El Niño (La Niña) event is occurring when the SST anomaly on the east equatorial Pacific is greater (lesser) than $0.5(-0.5)$ degrees Celsius with respect to the average value. The SST anomalies are related to the thermocline depth anomalies. Thus, the key quantities that characterize ENSO in the equatorial Pacific Ocean are the SST anomaly $\left(T_{E}\right)$ in the eastern part of the domain, usually described in terms of the Niño 3index (average SST in the area $5^{\circ} \mathrm{S}-5^{\circ} \mathrm{N}, 150^{\circ} \mathrm{W}-90^{\circ} \mathrm{W}$ ), and the zonal average of the anomaly of the thermocline depth $(h)$.

Exploiting these very peculiar physical conditions of the equatorial surface layer of the Pacific Ocean and from two-box (east and west side, respectively), two-strip (equatorial and off equatorial, respectively) approximations of the shallow water Navier-Stokes (NS) equations applied to the tropical Pacific Ocean, Jin obtained the following very simple system of linear equations for the Pacific equatorial thermocline depth anomaly $[10,11]$ :

$$
\begin{aligned}
& \dot{h}_{W}=-r h_{W}-\alpha \tau_{s} \\
& h_{E}-h_{W}=\tau_{s}
\end{aligned}
$$

where $\tau_{s}$ is the zonally-integrated wind stress anomaly, the subscript $W(E)$ means that the variables refer to the west (east) side of the basin, the friction term $r$ collectively represents the damping of the upper ocean system through mixing and the equatorial energy loss to the boundary layer currents at the east and west sides of the ocean basin, and it is set in the range 6.3 month $\lesssim r^{-1} \lesssim 8.0$ months $[10,11]$. Equation (1b) describes the zonal tilt of the thermocline, which is in balance with the equatorial zonal 
wind stress forcing on interannual time scales (e.g., [10,51]). The Kelvin and Rossby waves, which accomplish the adjustment processes, are filtered out, in this picture, because the timescale of the mode retained in this approximation is much longer than the basin crossing time of these waves. If one assumes that for a given steady wind stress forcing, the zonal mean thermocline anomaly of this linear system is about zero at the equilibrium state, that is, $h_{E}+h_{W}=0$ (at equilibrium), then from Equation (1), one finds that $\alpha$ shall be about half of $r$.

The work in $[10,11]$ provided a physical basis for the simple linear relationships in Equation (1), in the long wave, two-strip approximation.

Notice that if the wind stress $\tau_{s}$ were a fast forcing completely independent of the dynamics of the thermocline depth anomaly, Equation (1) would give rise to a very simple relaxation process with relaxation time given by $1 / r$, which, of course, is not what happens in reality. In fact, as we shall see below, the wind dynamics has a slow part that is strongly correlated with the dynamics of $h$ via a direct linear relationship with the other ENSO variable: the SST anomaly $T_{E}$.

A linear ODE for the thermodynamics relationship between the variation of the anomalous SST and the thermocline depth was proposed in $[10,11,52,53]$ and related subsequent works:

$$
\dot{T}_{E}=-c T_{E}+\gamma h_{E}+\delta_{S} \tau_{s, E}
$$

where the first term on the right-hand side is a damping process with a collective damping rate $c$. The second term describes the influence on $T_{E}$ of the thermocline displacement, and $\delta_{S}$ is the Ekman pumping coefficient of the vertical advective feedback processes due to the wind stress averaged over the domain, which actually can be considered vanishing (see $[10,52,53]$ for details): $\delta_{S}=0$.

Using Equation (1b), we can rewrite Equation (2) as:

$$
\dot{T}_{E}=-c T_{E}+\gamma h_{W}+\gamma \tau_{s}
$$

The above equation relates the dynamics of $T_{E}$ to that of $h_{W}$. However, as we have already observed, also the dynamics of $h_{W}$, given in Equation (1), depends on the values of the $T_{E}$ variable. This dependence is due to the fact that the wind stress $\tau_{s}$ is strongly influenced by the zonal SST gradient. In effect, in most of the works on the ROM [52,53] and on the DO [18], it is explicitly assumed that the following linear relationship holds true:

$$
\tau_{s}=b T_{E}+g(t)
$$

where $g(t)$ is a fast "chaotic" fluctuating function of time. In this work, we define as "chaotic" a unidimensional function of time $g(t)$ if its dynamics can be considered "almost " random so as to allow us to substitute it safely with a genuine stochastic process or to use a second-order perturbation projection approach, as hereafter specified. In the first case, we should exploit well-known works about limit theorems (e.g., [54]) and introduce a more specific chaotic hypothesis (e.g., [55,56]) that, actually, has been proven to be satisfied only by highly idealized mathematical models. In the second case, the requests are less stringent, because it is enough that for any $n \in \mathbb{N}$, the correlation function $\left\langle g(t) g\left(t+t_{1}\right) \ldots g\left(t+t_{n}\right)\right\rangle$ decays "quickly enough" for each $1 \leq k \leq n$, to avoid divergences in the neglected terms obtained with the perturbation procedure [29,57-59]. The extensive use of the quotes is to stress the fact that, as already stated in the Introduction, it is beyond the focus of the present work to discuss the dynamical foundation of stochastic processes/statistical mechanics. For further use, we specify that the symbol $\langle\ldots\rangle$ indicates a time average: $\langle a\rangle:=\lim _{\mathcal{T} \rightarrow \infty} \int_{0}^{\mathcal{T}} a(t) \mathrm{d} t / \mathcal{T}$, but we shall exploit the ergodic assumption and the Gibbs idea to turn the time averages into ensemble averages.

Equation (4) implies that the slow component of the wind stress and the slow component of $T_{E}$ are proportional to each other. Figure 1 of the paper [60] supports this assumption, and we further validate this hypothesis by analyzing the NOAA data [61], as detailed in Appendix A, where the wind stress anomaly $\tau_{s}$ is divided into slow $\left(\tau_{s, \text { slow }}\right)$ and fast $\left(\tau_{s, \text { fast }}\right)$ components. The $\tau_{s, \text { slow }}$ data are 
obtained from the one-year average of $\tau_{s}$ data, while $\tau_{s, \text { fast }} \equiv \tau_{s}-\tau_{s, \text { slow }}$ can be identified with $g(t)$ of (4).

Exploiting Equation (4), from Equations (1b) and (3), we obtain the well-known linear ROM:

$$
\begin{gathered}
\dot{h}_{W}=-r h_{W}-\alpha b T_{E}-\alpha g(t) \\
\dot{T}_{E}=\gamma h_{W}-\lambda T_{E}+\gamma g(t),
\end{gathered}
$$

with $\lambda \equiv c-\gamma b$.

Considering that $\alpha$ is quite smaller than $\gamma[10,11]$, we disregard the forcing term $-\alpha g(t)$ in the first equation of System (5), and finally, we are led to the following simplified linear ROM:

$$
\begin{aligned}
\dot{h}_{W} & =-r h_{W}-\alpha b T_{E} \\
\dot{T}_{E} & =\gamma h_{W}-\lambda T_{E}+g(t)
\end{aligned}
$$

where we have included the constant $\gamma$ in the definition of the fast part $g(t)$ of the wind stress $\tau_{s}$. The result of Equation (6) is really remarkable because it is a very simple, but still informative, description of a complex large-scale ocean/atmosphere process. We can consider it on the same footing as the Onsager linear regression principle, chemical reaction rate models [43] and other linear results of standard thermodynamics and statistical mechanics, which arise as large time and space scale phenomena from an underlying complex fast and chaotic dynamics.

\section{Is a Possible Internal Nonlinearity Relevant?}

Because of the linear relationship between $h_{E}$ and $T_{E}$ of Equation (2), the "internal" dynamics (i.e., for $g(t)=0$ ) of the ROM of Equation (6) is, by construction, linear. However, a linear relationship in the real world is almost always an approximation; thus in this section, we analyze the possibility that a nonlinear correction is effectively present in the interaction between the thermocline depth anomaly and the SST anomaly, and we try to evaluate if this correction can contribute to the non-Gaussian features of the ENSO. Actually, in [18], it is argued that a nonlinear cubic relationship between the anomalous subsurface temperature $T_{s u b}$ and the thermocline depth anomaly should be taken into account:

$$
T_{\text {sub }}=a h_{E}-e^{*} h_{E}^{3}+O\left(h_{E}^{5}\right),
$$

where the values for the coefficients $a$ and $e^{*}$ have been roughly estimated in [18] as $a \sim 0.14{ }^{\circ} \mathrm{Cm}^{-1}$ and $e^{*} \sim 3 \times 10^{-5}{ }^{\circ} \mathrm{C} \mathrm{m}^{-3}$. From this nonlinear relation, a small cubic term in $h_{E}$ would enter also in Equation (2) [18]. Given the very small value of the ratio $e^{*} / a$, the cubic term should play some role only for $h_{E} \geq \pm 2 \sigma_{h_{E}}\left(\sigma_{h_{E}}\right.$ is the standard deviation of $h_{E}$ that is a little over $10 \mathrm{~m}$ [62]), reaching the same value of the linear term for about $h_{E} \sim \sqrt{a / e^{*}}=36 \mathrm{~m}$ (about $3 \sigma_{h_{E}}$ ). Using these arguments, in [63], it has been already shown that assuming a nonlinear ROM linearly interacting with the atmosphere, we obtain a non-normal stationary PDF. However, as we can see in Figure. 5 of [60], both observations and numerical results show that the relationship between $T_{s u b}$ and $h$ is far more linear than what was suggested in [18]. In fact, this figure shows a small dispersion of the data around the linear fit, for a range of values of $h_{E}$ that spans beyond $\pm 4 \sigma_{h_{E}}$. Thus, we have evidence, from observations, that the nonlinear correction to the linear ROM is quite smaller that the (already small) value estimated in [18]. However, to evaluate the relevance of this small nonlinear term for the ENSO statistics, we also have to account for the behavior of the PDF for large values of $h$. To estimate the PDF including the nonlinear correction in the interaction between the thermocline depth anomaly and the SST anomaly, we consider the following nonlinear ROM:

$$
\begin{aligned}
& \dot{h}_{W}=-r h_{W}-\alpha b T_{E} \\
& \dot{T}_{E}=\gamma U^{\prime}\left(h_{W}\right)-\lambda T_{E}+g(t),
\end{aligned}
$$


in which

$$
U\left(h_{W}\right)=h_{W}^{2} / 2+\kappa h_{W}^{4} / 4+O\left(h_{W}^{6}\right)
$$

where for a generic function $f(x)$, we have defined $f^{\prime}(x):=d f(x) / d x$ and $\kappa$ is a parameter that, according to the above results, is much smaller than the value of the ratio $e^{*} / a$, i.e., $\kappa<<10^{-4} \mathrm{~m}^{-2}$. The stationary PDF that can be derived from Equation (8), considering $g(t)$ as an additive fast fluctuating forcing, is as follows. We change variables: $v \equiv-r h_{W}-\alpha b T_{E}, x=h_{W}$, and Equation (8) becomes:

$$
\begin{aligned}
& \dot{x}=v \\
& \dot{v}=-\alpha b \gamma U^{\prime}(x)-\lambda r x-(r+\lambda) v-\alpha b g(t) .
\end{aligned}
$$

If $g(t)$ is assumed to be a white noise with a correlation function $\langle g(0) g(t)\rangle=2 d \delta(t)$, Equation (10) describes the dynamics of a particle with coordinate $x$, velocity $v$, in an "almost" harmonic potential given by:

$$
V(x)=\alpha b \gamma U(x)+\lambda r \frac{x^{2}}{2} \simeq(\lambda r+\alpha b \gamma) \frac{x^{2}}{2}+\alpha b \gamma \kappa \frac{x^{4}}{4}
$$

and interacting with a thermal bath with friction $r+\lambda$ and "temperature" $\Theta=\alpha^{2} b^{2} d /(r+\lambda)$. It is well known that the stationary PDF for such a system is the "canonical" one:

$$
\rho_{s}(x, v)=Z e^{-\frac{H(x, v)}{\Theta}},
$$

where $\mathrm{Z}$ is the normalization constant and $H(x, v) \equiv V(x)+\frac{v^{2}}{2}$ is the Hamiltonian. Thus, $\Theta$ is the variance of the "velocity" variable $v: \Theta=\left\langle v^{2}\right\rangle_{s} \equiv \sigma_{v}^{2}$. As for the $x\left(=h_{W}\right)$ variable, we notice that, given the small value of the $\kappa$ parameter, the non-harmonic term $\alpha b \gamma \kappa x^{4} / 4$ in the potential $V(x)$ of Equation (11) gives a negligible contribution to the variance; thus, we also have $\Theta \simeq(\lambda r+\alpha b \gamma)\left\langle x^{2}\right\rangle_{s}:=$ $(\lambda r+\alpha b \gamma) \sigma_{x}^{2}$. Therefore, the stationary PDF of Equation (12) is very well approximated by a Gaussian function until the nonlinear term of the potential $\alpha b \gamma \kappa \frac{x^{4}}{4}$ enters into play, but, as already noted, this happens when $x=h_{W}$ far exceeds at least two standard deviations, i.e., where the non-Gaussian feature could not really emerge because of the extremely low value of the PDF of Equation (12). Thus, we are led to the conclusion that the possibly nonlinear interaction between the internal ROM variables cannot inherently be the cause of the observed non-Gaussian properties of the ENSO statistics.

Notice that if we consider the external forcing $g(t)$ as a correlated noise (i.e., not white) or as a weak deterministic chaotic perturbation with a finite time scale (namely, with correlation function that decays in a finite time), using a Zwanzig-like projection approach in the perturbation version of [64-66], we obtain a stationary PDF for the ROM variables that is similar to the one in Equation (12), apart from some small changes in the parameter $\Theta$ and in the coefficients of the potential $V(x)$. Thus, also in these non-Markovian cases, the same argument of the previous white noise case applies: the non-Gaussian features of the PDF would be negligible and not as pronounced as observed.

So far, we have shown that analyzing the relations between the dynamics of $h_{W}$ and $T_{E}$, it is hard to find nonlinearities strong enough to justify the non-Gaussian behavior of the ENSO statistics. Thus, from now on, we shall assume that the non-harmonic term in the potential $U\left(h_{W}\right)$ of Equation (9) can be neglected. Namely, we return to the linear ROM of Equation (6) and attempt to justify the observed nonlinearities focusing on the interaction of the slow components of the ROM of Equation (6) with $g(t)$.

It is worthwhile to remark that a recent study [67] has highlighted the importance of the nonlinear advective terms in the upper-ocean temperature equations as a source of ENSO nonlinearities and the resulting non-Gaussianity of eastern Pacific SST anomalies. These nonlinear advective terms, which have been collectively termed Nonlinear Dynamical Heating (NDH), are primarily controlled by the anomalies of the zonal advection term $\left(u^{\prime} \partial_{x} T^{\prime}\right.$; see [67] for the nomenclature). However, the asymmetric influence of the nonlinear zonal advection term upon warm and cold ENSO events 
may be linked to the distinctive multiplicative character of the anomalous wind forcing of ENSO, which is stronger for positive than negative anomalies. Nonlinearities in the surface forcing and their influence on the SST statistics are described in the next section.

Before doing that, however, we simplify Equation (6) a little in the following way:

$$
\begin{aligned}
\dot{h} & =-\omega T_{E} \\
\dot{T}_{E} & =\omega h-\lambda T_{E}+g(t),
\end{aligned}
$$

where $h \equiv\left(h_{W}+h_{E}\right) / 2$ is the zonally-averaged thermocline depth anomaly (directly related to the anomalous heat accumulated in the Equatorial Pacific Ocean), a choice justified by the arguments and results of [12], shortly summarized hereafter. First, Equation (1b) implies that the tilt of the thermocline reacts almost instantaneously to wind stress, but it is more realistic to assume that the adjustment of the thermocline depth takes a finite time, approximately the time it takes for a Kelvin wave to propagate from the western central Pacific to the east. Thus, Equation (1b) should be replaced by:

$$
\dot{h}_{E}=-\gamma_{h}\left(h_{E}-h_{W}-\tau_{s}\right) \text {. }
$$

Second, repeating the same steps that led us from Equation (1b) to Equation (6), we get an unperturbed ROM given by three differential equations that better represent the ENSO dynamics, leading to a better agreement among the parameter of the model and observations. Finally, studying in detail the feature of this linear three-degrees of freedom system, we see that one eigenstate has a fast decay time (shorter than one month); thus, the dynamics is attracted toward a two-dimensional slow manifold, well represented by the reduced ROM of Equation (13).

Suitable values for $\omega$ and $\lambda$ (the "friction" coefficient) can be obtained by using phenomenological considerations when deriving the ROM from building block equations and/or directly from a fit to observations (see [12]). In the literature, we find different values that range from $2 \pi / 48$ month $^{-1}-2 \pi$ $/ 36$ month $^{-1}$ for $\omega$ and from $1 / 12$ month $^{-1}-1 / 6$ month $^{-1}$ for $\lambda$. We shall see in the following that we can shrink these ranges.

\section{The Multiplicative Nature of the Forcing}

In some recent works, it has been shown that to account for the instability of the dynamics, the skewness and the tail of the observed stationary PDF of the ENSO, the perturbation forcing $g(t)$ of the ROM cannot be simply additive, but a multiplicative term, directly correlated with the additive one, should also be considered $[14,15,17]$, leading to a perturbation of the form:

$$
g(t)=\epsilon\left(1+\beta T_{E}\right) \xi(t),
$$

where $\xi(t)$ is a fast chaotic fluctuating term and $\epsilon$ is a parameter that controls the strength of the interaction. Many facts support the hypothesis given by Equation (14):

- the multiplicative nature of the ENSO forcing provided by the Madden-Julian Oscillation (MJO) and its higher frequency tail $([68,69]$ and the references therein);

- multiplicative fast forcings typically emerge from the general perturbation approaches to large-scale ocean dynamics [70,71];

- considering the nonlinear nature of the heat budget equation for the surface mixed layer [72], we get multiplicative fast fluctuations in both the net surface/subsurface heat flux and the advective contributions $[16,67,70,71]$.

Concerning the last point, we again notice that the equatorial Pacific zonal subsurface NDH $u^{\prime} \partial_{x} T^{\prime}$, which plays a role in the asymmetry of the El Niño-La Niña events [67], may be linked to the multiplicative term $\epsilon \beta T_{E} \xi(t)$ of Equation (14), assuming that $\xi(t)$ and $T_{E}$ are directly related to $u^{\prime}$ and $\partial_{x} T^{\prime}$, respectively.

Thus, inserting Equation (14) into the ROM of Equation (13), we get: 


$$
\begin{aligned}
\dot{h} & =-\omega T_{E} \\
\dot{T}_{E} & =\omega h-\lambda T_{E}+\epsilon\left(1+\beta T_{E}\right) \xi(t),
\end{aligned}
$$

that is the final model we shall take into account hereafter.

When $\xi(t)$ is a white noise, the term $\left(1+\beta T_{E}\right) \xi(t)$ is often named Correlated-Additive-Multiplicative (CAM) noise [21-23], but we shall extend this notation also to deterministic (i.e., not stochastic) processes, saying that $\left(1+\beta T_{E}\right) \xi(t)$ is a CAM forcing. In Section 6, starting from observations, we shall use a statistical inference method for diffusion processes with nonlinear drift to estimate the effective contribution of the CAM forcing with respect to a more simple additive fast forcing.

In general, it is reasonable that at least a part of the additive forcing should not be correlated with the multiplicative one, i.e., that there are different independent fast chaotic forcings that can be collected as an independent additive contribution, uncorrelated to the previous one, considered above:

$$
g(t)=f(t)+\left(1+\beta T_{E}\right) \xi(t)
$$

where $f(t)$ is uncorrelated with $\xi(t)$. However, for the sake of simplicity, we do not consider this possibility here. This more general ROM will be examined in a subsequent paper.

We want to stress again that we do not make the assumption that the forcing term $\xi(t)$ of Equation (15) has a stochastic nature. $\xi(t)$ is a deterministic "chaotic" forcing, and statistics is introduced by using the Gibbs concept of ensemble (our PDF).

\section{The Fokker-Planck Equation Guiding the Statistics of the ROM}

The ROM of Equation (15) must be validated by observations of the ENSO, namely we must use some data analysis approach to infer the values of the coefficients of the ROM of Equation (15). Due to the multiplicative character of the perturbation, the system is not linear; thus, strictly speaking, well consolidated approaches based on the Gaussian properties of the data dispersion, such as the Linear Inversion Model (LIM), cannot be used in this case. However, as will be shown in the next section, if we observe only, or if we are interested only in the first (the mean) and the second (the variance) moments, then the system of Equation (15) looks to be linear, with eigenvalues that are weakly renormalized by the $\beta$ parameter. Of course, replacing the nonlinear ROM of Equation (15) with a linear one, all the information concerning large deviations from the averages will be lost. Because we are interested in evaluating the role of the nonlinearities in the ENSO dynamics, modeled by the perturbed ROM, we would need an FPE for the PDF of the SST anomalies that fully retains the nonlinear nature of the interaction between the ENSO variables of interest $\left(h, T_{E}\right)$ and the collective variable $\xi(t)$ of Equation (15). In this way, in fact, a comparison between the FPE predictions and observations should possibly validate the model and allow one to estimate the relative weight of the multiplicative perturbation. In this section, we briefly recall and use some of the results of $[17,26,27,32,33]$ to introduce an explicit FPE for the PDF of the ROM system.

To be really in a position to distinguish, in the model of Equation (15), between the dynamics of the ENSO variables $\left(h, T_{E}\right)$ and that of the perturbation $\xi(t)$, it is implicitly assumed that there is a time scale separation and/or a weak interaction between these two subsystems. It is a fact that the dynamics of the atmosphere forcing triggering the ENSO, which is represented by the term proportional to the $\epsilon$ parameter in Equation (15), has a time scale that is shorter than the time scale of the average dynamics of the ENSO. Thus, we can say that the autocorrelation time $\tau$ of $\xi(t)$ is smaller than the period and relaxation time of the unperturbed ROM, given by $1 / \sqrt{\omega^{2}-\lambda^{2} / 4}$ and $2 / \lambda$, respectively. Notice that "shorter" does not necessarily mean "extremely shorter", but if this is the case, under some conditions about the features of the dynamics of the $\xi$ variable, we can approximate $\xi(t)$ by a white noise $[28,55,56,73]$, with $\langle\xi(0) \xi(t)\rangle=2\left\langle\xi^{2}\right\rangle \tau \delta(t)$. It is a well-known result that if $\xi(t)$ is replaced with a white noise, Equation (15) becomes equivalent to the following Fokker-Planck Equation (FPE) for the Probability Density Function (PDF) $\sigma(h, T ; t)$ of the ROM variables (we shall use the Stratonovich 
integration rule for white noise dynamics [74]; hereafter, for the sake of simplicity, we shall use $T$ for $T_{E}$ and $\partial_{t} \equiv \partial / \partial t, \partial_{h} \equiv \partial / \partial h$ and $\left.\partial_{T} \equiv \partial / \partial T\right)$ :

$$
\begin{aligned}
\partial_{t} \sigma(h, T ; t)= & \left\{\omega \partial_{h} T-\omega \partial_{T} h+\lambda \partial_{T} T+D \beta \partial_{T}(1+\beta T)\right. \\
& \left.+\partial_{T} D(1+\beta T)^{2} \partial_{T}\right\} \sigma(h, T ; t) \\
= & \left\{\omega \partial_{h} T-\omega \partial_{T} h+\lambda \partial_{T} T-D \beta \partial_{T}(1+\beta T)\right. \\
& \left.+\partial_{T}^{2} D(1+\beta T)^{2}\right\} \sigma(h, T ; t)
\end{aligned}
$$

where $D \equiv \epsilon^{2}\left\langle\xi^{2}\right\rangle \tau$ is the "standard" diffusion coefficient.

In the case where the time scale separation between the unperturbed ROM and the fast forcing is not so extreme, as we have already stressed, we cannot approximate the deterministic forcing $\xi(t)$ with a white noise, and we have to work with ensembles (i.e., PDF) for which the time evolution is guided by the Liouvillian corresponding to the equation of motion of the perturbed ROM of Equation (15). Starting from the Liouville equation, assuming weak perturbation of the ROM (small $\epsilon$ values) and using a projection perturbation approach, it is thus possible to derive an effective FPE that well approximates the dynamics of the PDF [17] (see also Appendix B for a short summary of the projection procedure, adapted to the present case of Equation (15)):

$$
\begin{aligned}
& \partial_{t} \sigma(h, T ; t)=\left\{\omega \partial_{h} T-\omega \partial_{T} h\right. \\
& +\left(\lambda+D \beta^{2}\right) \partial_{T} T+D \beta \partial_{T} \\
& \left.+\partial_{T} A(h, T) \partial_{T}+\partial_{T} B(h, T) \partial_{h}\right\} \sigma(h, T ; t),
\end{aligned}
$$

where the decay time $\tau$ of the auto-correlation function of $\xi$ is defined in the following way: if $\varphi(t) \equiv\langle\xi(t) \xi(0)\rangle /\left\langle\xi^{2}\right\rangle$ is the normalized autocorrelation function of $\xi$, then $\tau \equiv \int_{0}^{\infty} \varphi(t) \mathrm{d} t$. As in [17], the diffusion functions $A(h, T)$ and $B(h, T)$ are second order polynomials of the ROM variables $(h, T)$ :

$$
\begin{aligned}
A(h, T)= & A_{0}+\beta A_{1} h+\beta A_{2} T \\
& +\beta^{2} A_{3} h T+\beta^{2} A_{4} T^{2} \\
B(h, T)= & B_{0}+\beta B_{1} h+\beta B_{2} T \\
& +\beta^{2} B_{3} h T+\beta^{2} B_{4} T^{2} .
\end{aligned}
$$

As is shown in the Appendix $\mathrm{B}$, the $A_{i}$ and $B_{i}$ coefficients are proportional to $\epsilon^{2}$, do not depend on the $\beta$ parameter and are linear combinations of the Fourier transform of the function $\varphi(t)$, evaluated at the frequencies $2 \Omega$ and $\Omega-i \lambda / 2$, where $\Omega \equiv \sqrt{\omega^{2}-\lambda^{2} / 4}$ is the effective frequency of the unperturbed ROM.

From the FPE, it is possible to get all the relevant statistical information about the ENSO variables, including the $T$ moments and the stationary PDF. From a formal point of view, the FPE in Equation (18) is a second order Partial Differential Equation (PDE) with the discriminant [75] equal to $B(h, T)^{2} / 2 \geq 0$; thus, for non-vanishing $B(h, T)$, this PDE is hyperbolic, and not a parabolic one like the FPE of Equation (17) derived from a "true" stochastic Markovian process. The diffusion coefficient $B(h, T)$ is a signature of the finite (i.e., "non infinite") time scale separation between the dynamics of the system of interest and that of the booster.

\section{Inference of the Statistical Features of the ROM from Observations}

\subsection{Linear Equation of Motion for the First Two Moments of the ROM and the White Noise Approximation}

Using the FPE of Equations (18) and (19), it is easy to obtain a set of closed equations of motion for the first $n$-th moments of the ROM. For the moments up to the second ones, we get: 


$$
\begin{aligned}
\langle\dot{h}\rangle & =-\omega\langle T\rangle \\
\langle\dot{T}\rangle & =\left(\omega+\beta^{2} A_{3}\right)\langle h\rangle-\left(\lambda-\beta^{2} A_{4}\right)\langle T\rangle+\beta A_{0} \\
\left\langle\dot{h^{2}}\right\rangle & =-2 \omega\langle h T\rangle \\
\langle\dot{h} T\rangle & =\left(\omega+\beta^{2} A_{3}\right)\left\langle h^{2}\right\rangle \\
& -\left(\lambda-\beta^{2} D\right)\langle h T\rangle-\left(\omega-\beta^{2} B_{4}\right)\left\langle T^{2}\right\rangle \\
& +\beta\left(D+A_{0}-A_{4}\right)\langle h\rangle-\beta B_{2}\langle T\rangle+B_{0} \\
\left\langle\dot{T}^{2}\right\rangle & =2\left(\omega+2 \beta^{2} A_{3}\right)\langle h T\rangle-2\left(\lambda-\beta^{2} 2 A_{4}\right)\left\langle T^{2}\right\rangle \\
& +2 \beta\left(2 A_{0}+A_{4}\right)\langle T\rangle+2 \beta A_{3}\langle h\rangle+2 A_{0},
\end{aligned}
$$

from which (the subscript " $s$ " stands for "stationary"):

$$
\begin{aligned}
\langle T\rangle_{s} & =0 \\
\langle h\rangle_{s} & =-\frac{\beta A_{0}}{\beta^{2} A_{3}+\omega} \approx-\beta A_{0}\left(\omega-\beta^{2} A_{3}\right)+O\left(\epsilon^{4}\right) \\
\left\langle T^{2}\right\rangle_{s} & =\frac{A_{0}}{\lambda-2 \beta^{2} A_{4}}\left(1-\frac{\beta^{2} A_{3}}{\beta^{2} A_{3}+\omega}\right) \\
& \approx \frac{A_{0}}{\lambda}\left[1+\beta^{2}\left(2 \frac{A_{4}}{\lambda}-\frac{A_{3}}{\omega}\right)\right]+O\left(\epsilon^{6}\right) \approx \frac{A_{0}}{\lambda}+O\left(\epsilon^{4}\right) \\
\left\langle h^{2}\right\rangle_{s}-\langle h\rangle_{s}^{2} & =\left\langle T^{2}\right\rangle_{s}
\end{aligned}
$$

Thus, the variance of the stationary PDF can be considered independent of $\beta$.

Equation (20a) is equivalent to the equation of motion of a linear dissipative oscillator, with bare frequency $\sqrt{\omega^{2}+\beta^{2} A_{3}}$ and friction coefficient $\left(\lambda-\beta^{2} A_{4}\right)$, perturbed by the constant force $\beta A_{0}$. The weak perturbation assumption (small $\epsilon$ ), together with the fact that $\beta<<1$ (see below and [17]) imply that $\omega+\beta^{2} A_{3} \sim \omega$ and $\left(\lambda-\beta^{2} A_{4}\right) \sim \lambda$. Thus, the equation of motion of the first moment (Equation (20a)) is very similar to that of the unperturbed ( $\epsilon=0)$ ROM (apart from the constant forcing $\beta A_{0}$ that can be disregarded, as we will show shortly). This fact is really important because from a physical point of view, it gives a sound meaning to the definition of the unperturbed $(g=0)$ ROM of Equation (13): it is the dynamical system that corresponds to the "slow" part of the ENSO dynamics.

Concerning the constant force $\beta A_{0}$ appearing in Equation (20a), it is not really measurable; in fact, it is an "artifact" of the final approximation that, from the ROM of Equation (6), leads to Equation (15). This is clear from the fact that this constant forcing yields a non-vanishing average stationary value for the thermocline depth anomaly: $\langle h\rangle_{s} \sim-\beta A_{0} / \omega$. To cure this flaw, we should replace $h$ with $h-\beta A_{0} / \omega$ in Equation (15) or we could add a weak friction term $-\lambda_{h} h$ to the first equation of the ROM model (15). However, because we shall focus our attention on $T$, we will leave the ROM unchanged.

Concerning the equations of motion of the second moments (Equation (20b)), we see that they weakly depend on the $B$ diffusion coefficient. Moreover, using the relationships of Equations (A10), (A14) and (A15), we have that they mainly depend on $\beta$ and $A_{0}$; thus, the dynamics of the second moments should not depend too much on the time scale separation between the average ROM dynamics and that of the fast forcing $\xi$. Estimates of the time scale $1 / \lambda$ are in the range of 6-12 months. Assuming an exponential decay of the autocorrelation function of $\xi$, i.e. $\varphi(t)=\exp (-t / \tau)$, we can verify that for $\tau$ up to $\sim 3$ months, the transport coefficient $A$ has the same structure it has in the limit of very large time scale separation $(\tau \rightarrow 0)$, namely $A(T) \sim A_{0}(1+\beta T)^{2}$ (see Figure 2 ), and as is shown in Figure 3, the dynamics of the second moments is almost independent of $\tau$. 


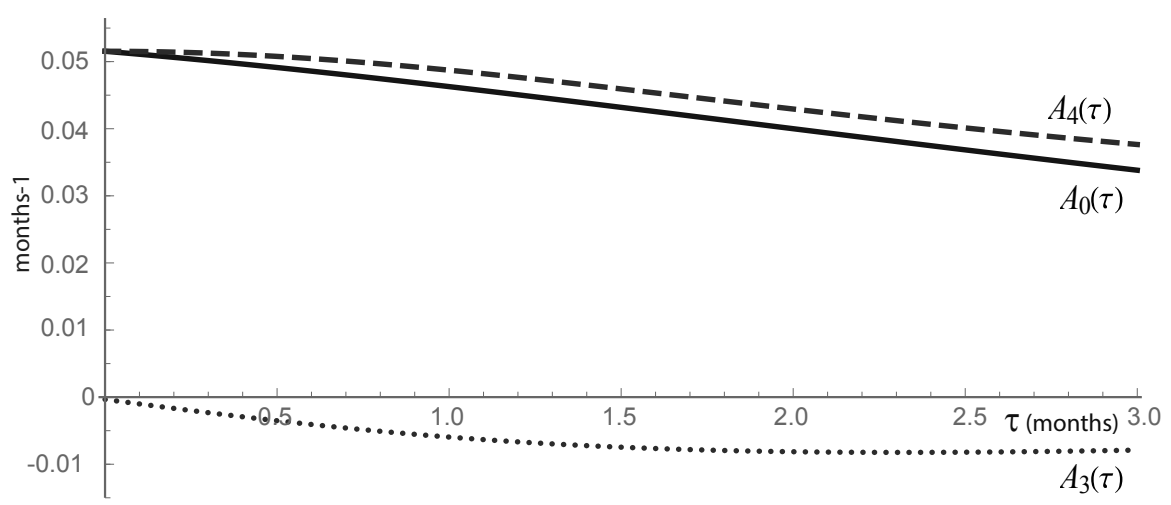

Figure 2. The values of the coefficients $A_{0}, A_{4}$ and $A_{3}$ vs. $\tau$ for $\varphi(t)=\exp (-t / \tau)$ for $1 / \lambda=12$ months. In the shown range 0 months $\leq \tau \leq 3$ months, $A_{4} \sim A_{0}$ and $A_{3} \sim 0$. We recall that $A_{2}=A_{4}+A_{0}$ and that $A_{1}=A_{3}$. Thus, $A \sim A_{0}(1+\beta T)^{2}$, which must be compared to $A=D(1+\beta T)^{2}$, which holds true in the limit of a very large time scale separation between the dynamics of the ROM and that of the booster (see the text for details).
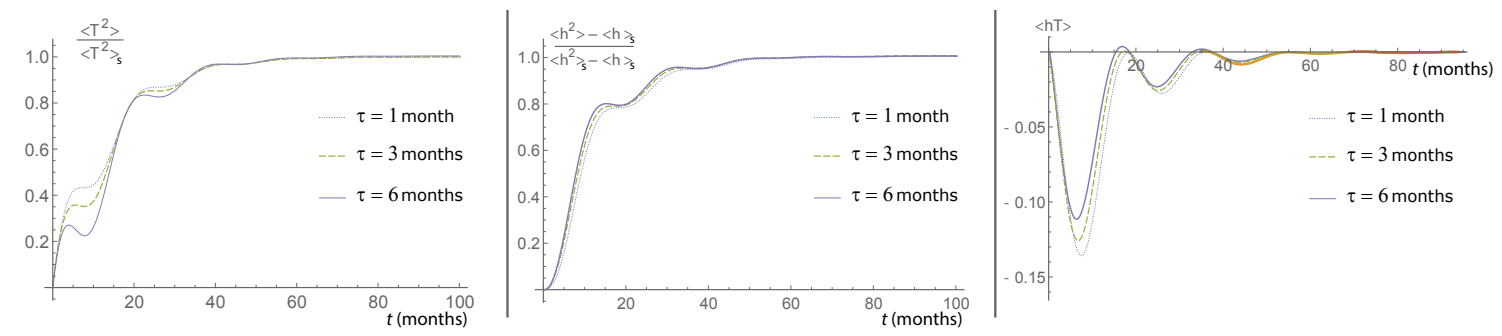

Figure 3. Evolution of the variance of $T$ (left), $h$ (center) and of the cross-correlation between $T$ and $h$, obtained from Equation (20b) with the $A_{i}$ and $B_{i}$ coefficients given by Equations (A7)-(A14), in the case where $1 / \lambda=12$ month and $\varphi(t)=\exp (-t / \tau)$, for different values of $\tau$. Solid line: $\tau=6$ months; dashed line: $\tau=3$ months; dotted line: $\tau=1$ month. Curves for different $\tau$ values are very similar.

The results of this section allow us to get two main important conclusions:

1. analyzing only the first and second moments/cumulants/cross-correlation functions of the observations data, we cannot identify/detect the (possible) nonlinearity due to the interaction with the atmosphere, in the ENSO system;

2. comparing the first and second moments/cumulants/cross-correlation functions, we obtain from the FPE of Equation (18) with observations, it would be really hard to determine how small the time scale $\tau$ of decaying of the correlation function of the effective noise perturbing the ROM is. On the other hand, as has been shown in [17], also for $\tau \rightarrow 0$, the FPE of Equation (18) well accounts for the non-Gaussianity of the ENSO statistics. Thus, for the sake of simplicity, from now on, we shall use the FPE of Equation (17), which is the white noise limit of Equation (18), even if we are aware of the fact that the time scale separation between the dynamics of the averaged ROM and that of the fast atmosphere is not so "extreme".

In the next subsection, we shall analyze Point 1 in detail.

\subsection{The Covariance Matrix of the ROM and the Comparison with the ENSO Data}

Given the results of the previous subsection, we assume that to describe the statistics of the ENSO, we can use the simplified FPE of Equation (17), instead of the more general Equation (18). This FPE must be validated with data from observations. 
To this end, we write again the first equations for the time evolution of the first two moments, using the FPE Equation (17) (we also use the shift $h \rightarrow h+\beta D / \omega$ to ensure that the mean value of the thermocline depth anomaly is zero):

$$
\begin{aligned}
\langle\dot{h}\rangle & =-\omega\langle T\rangle \\
\langle\dot{T}\rangle & =\omega\langle h\rangle-\left(\lambda-\beta^{2} D\right)\langle T\rangle \sim \omega\langle h\rangle-\lambda\langle T\rangle \\
\left\langle\dot{h^{2}}\right\rangle & =-2 \omega\langle h T\rangle \\
\langle h \bar{T}\rangle & =\omega\left\langle h^{2}\right\rangle-\left(\lambda-\beta^{2} D\right)\langle h T\rangle-\omega\left\langle T^{2}\right\rangle \\
& \sim \omega\left\langle h^{2}\right\rangle-\lambda\langle h T\rangle-\omega\left\langle T^{2}\right\rangle \\
\left\langle\dot{T}^{2}\right\rangle & =2 \omega\langle h T\rangle-2\left(\lambda-2 \beta^{2} D\right)\left\langle T^{2}\right\rangle+4 \beta D\langle T\rangle+2 D \\
& \sim 2 \omega\langle h T\rangle-2 \lambda\left\langle T^{2}\right\rangle+4 \beta D\langle T\rangle+2 D .
\end{aligned}
$$

For the sake of simplicity, in the above equations, we have explicitly made the approximation of neglecting the terms proportional to $\beta^{2} D$, assuming that $\epsilon \ll 1$ and $\beta \ll 1$. The validity of this assumption will be shown later. In any case, these approximations could be weakened without affecting the derivation below. From the above equations, we get the following stationary first and second moments:

$$
\begin{gathered}
\langle h\rangle_{s}=\langle T\rangle_{s}=0 \\
\left\langle h^{2}\right\rangle_{s}=\left\langle T^{2}\right\rangle_{s} \sim \frac{D}{\lambda} .
\end{gathered}
$$

The values of the ratio $D / \lambda$ can be obtained from the observations with a very good accuracy. According to Equation (24), $D / \lambda$ can be inferred from the variance $\sigma^{2}=\left\langle T^{2}\right\rangle_{s}$ of the Niño 3 time series, leading to $D / \lambda \sim 0.8$ (we recall that $D \equiv \epsilon^{2}\left\langle\xi^{2}\right\rangle \tau$ is the "standard" diffusion coefficient). For the other coefficients in the FPE of Equation (17), we focus our attention on the elements of the covariance matrix $\langle x(t) y(0)\rangle_{s}$, where " $x$ " and " $y$ " can be either $h$ or $T$. We note that by definition, we have:

$$
\langle x(t) y(0)\rangle_{s}=\left\langle\left(e^{\mathcal{L}_{F P E}^{+} x}\right) y\right\rangle_{s}
$$

where $\mathcal{L}_{F P E}^{+}$is the adjoint of the Liouvillian defined by the FPE of Equation (17). Thus, the evolutions $\left(e^{\mathcal{L}_{F P E}^{+} t} h\right)$ and $\left(e^{\mathcal{L}_{F P E}^{+} t} T\right)$ are solutions of the differential equations for the lowest order moments of Equation (23a). Because Equation (23a) is linear, the solutions will be linear combinations of $h$ and $T$ :

$$
\begin{aligned}
& \left(e^{\mathcal{L}_{F P E}^{+} h}\right)=a_{h, h}(t) h+a_{h, T}(t) T \\
& \left(e^{\mathcal{L}_{F P E}^{+} T}\right)=a_{T, h}(t) h+a_{T, T}(t) T
\end{aligned}
$$

where:

$$
\begin{aligned}
& a_{h, h}(t)=e^{-\frac{\lambda}{2} u}\left[\cos (\Omega u)+\frac{\lambda}{2} \frac{\sin (\Omega u)}{\Omega}\right] \\
& a_{h, T}(t)=-e^{-\frac{\lambda}{2} u} \omega \frac{\sin (\Omega u)}{\Omega} \\
& a_{T, h}(t)=-a_{h, T}(t) \\
& a_{T, T}(t)=e^{-\frac{\lambda}{2} u}\left[\cos (\Omega u)-\frac{\lambda}{2} \frac{\sin (\Omega u)}{\Omega}\right] .
\end{aligned}
$$


Exploiting Equation (26), Equation (25) becomes:

$$
\begin{aligned}
\langle h(t) h(0)\rangle_{s} & =\left\langle h^{2}\right\rangle_{s} a_{h, h}(t) \\
\langle T(t) h(0)\rangle_{s} & =\left\langle h^{2}\right\rangle_{s} a_{T, h}(t) \\
\langle h(t) T(0)\rangle_{s} & =\left\langle T^{2}\right\rangle_{s} a_{h, T}(t) \\
\langle T(t) T(0)\rangle_{s} & =\left\langle T^{2}\right\rangle_{s} a_{T, T}(t)
\end{aligned}
$$

where (see Equation (24)) $\left\langle T^{2}\right\rangle_{s}=\left\langle h^{2}\right\rangle_{s} \sim D / \lambda \sim 0.8 \pm 0.3$. In Figure 4, we show the time evolution of the elements of the correlation matrix obtained from observations and the best fit obtained with Equation (27). This best fit yields values of $\omega=2 \pi / 48 \mathrm{month}^{-1}$ and $\lambda=1 / 10 \mathrm{month}^{-1}$, but the residuals of these fitting functions are quite large. For that, in the same figure, we also insert the plot of the same functions of Equation (27), but with the ROM coefficients given by $\omega=2 \pi / 48$ month $^{-1}$ and $\lambda=1 / 12$ month $^{-1}$, which, as we shall see later, well agree also with other aspects of the data analysis.

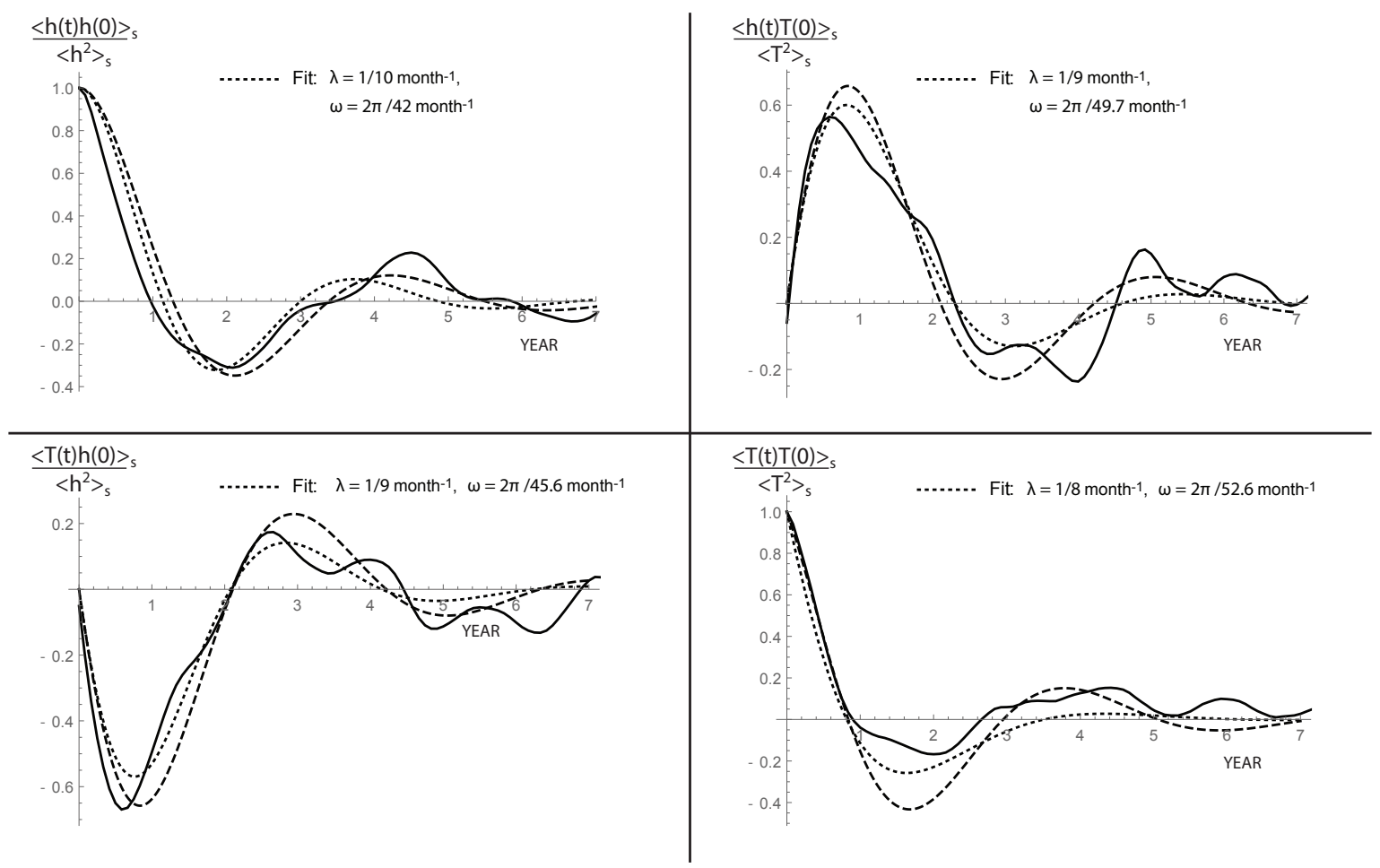

Figure 4. Correlation matrix $\langle x(t) y(0)\rangle_{s} /\left\langle y^{2}\right\rangle_{s}$, where " $x$ " and " $y$ " can be either $h$ or $T$. Solid line: from the NOAA data (the $h$ values are taken from [76] and refer to the anomalies of the Volume of Warm Water $(\mathrm{WWV})$ in the basin $120^{\circ} \mathrm{E}-80^{\circ} \mathrm{W}, 5^{\circ} \mathrm{S}-5^{\circ} \mathrm{N}$, in the time range from January 1982-December 2017). Dashed line, from the Fokker-Planck Equation (FPE) of the Recharge Oscillator Model (ROM), i.e., from Equations (27) and (28) in the case where $\omega=2 \pi / 48$ month $^{-1}$ and $\lambda=1 / 12$ month $^{-1}$. Dotted line: a fit with the same functions of Equations (27) and (28).

After the above results, it is not surprising that also the power spectrum analysis of the Niño 3 data shown in Figure 5 does not allow us to infer the non-Gaussian character of the ENSO statistics. In fact, although in Figure 5, the power spectrum is evaluated directly as the periodogram of the observed Niño 3 index, it is well known that it is directly related to the Fourier transform of the autocorrelation function of $T$, which, in our ROM with CAM forcing, does not depend on $\beta$ (see Equations (27) and (28)). From a quantitative point of view, the comparison of the observed and theoretical spectra in Figure 5 confirms the already found values for the unperturbed ROM parameters: $\omega=2 \pi / 48 \mathrm{month}^{-1}$, $\lambda=1 / 10$ month $^{-1}$. 


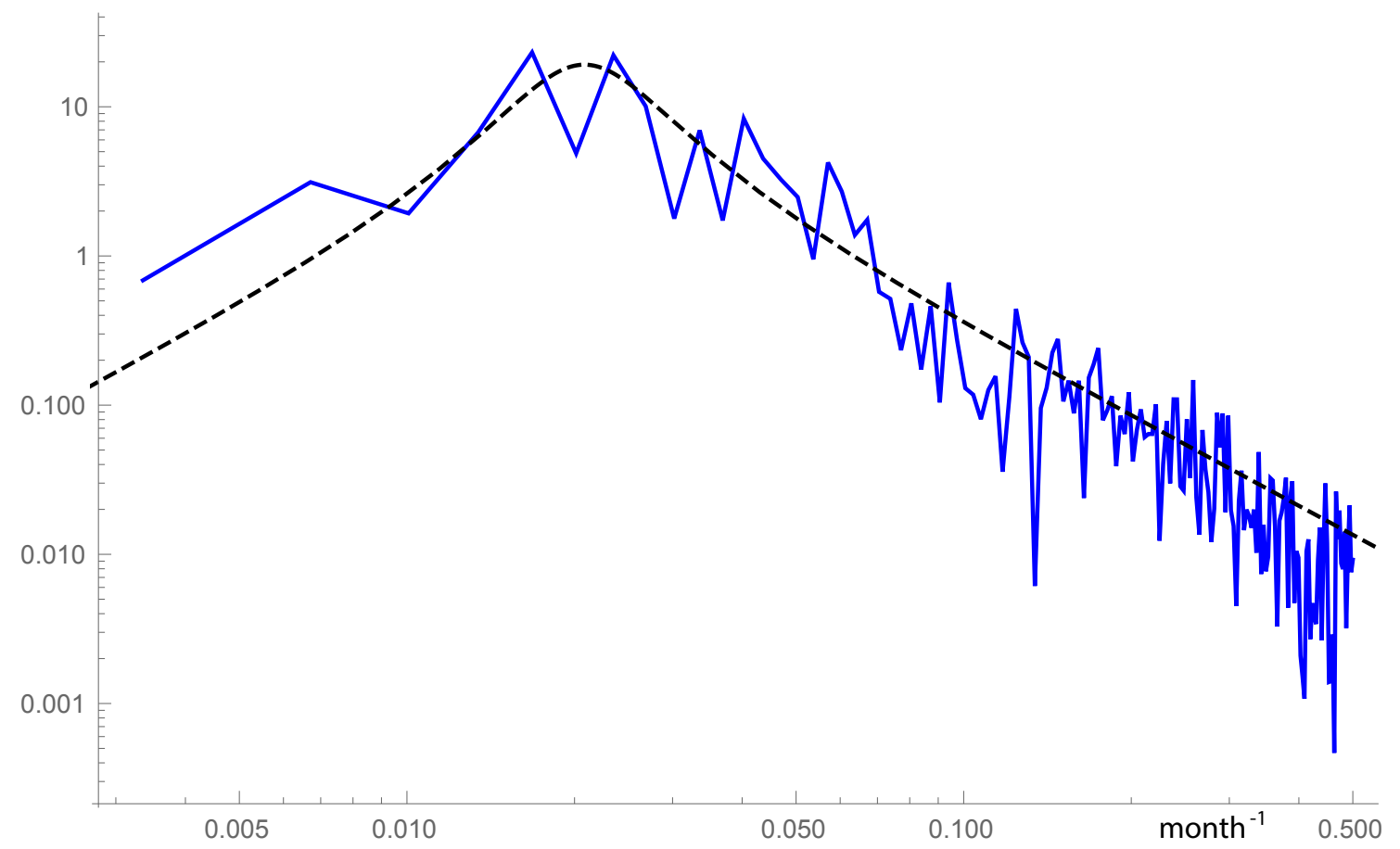

Figure 5. Solid blue line: periodogram of the Niño 3 data from NOAA evaluated averaging over non-overlapping partitions of length 300 months. Dashed black line: theoretical power spectrum of an ROM with $\omega=2 \pi / 48$ month $^{-1}, \lambda=1 / 10$ month $^{-1}$ and additive white noise perturbation.

\subsection{Signatures of a Nonlinear Perturbation: Skewed Stationary PDF and High Frequency of Strong Events}

As we have already noticed, if we focused on the first and second moments of the ENSO, we would not be able to estimate the value of the parameter $\beta$ responsible for the nonlinearity of the perturbed ROM of Equation (15) (or the FPE of Equation (17)). To infer the value of the $\beta$ parameter, we have to focus our attention on the non-Gaussian character of the ENSO statistics; hence, we have to evaluate the stationary PDF of the FPE of Equation (17). Although this FPE is simplified with respect to the more general one (Equation (18)), the exact stationary PDF is not known. It has been shown in [17], using a reasonable ansatz, that it is possible to obtain the following analytic expression for the reduced stationary PDF of the sole $T$ variable (see also Appendix C):

$$
\begin{gathered}
p_{s}(T)=\beta f_{\mu}\left(\frac{\mu-2}{1+\beta T}\right) \quad \text { for } T>-1 / \beta \\
p_{s}(T)=0 \quad \text { for } T \leq-1 / \beta, \\
\mu \equiv 1+\frac{\lambda}{D \beta^{2}},
\end{gathered}
$$

where the Gamma-like density function $f_{\mu}(x)$ is defined as:

$$
f_{\mu}(x) \equiv \frac{1}{(\mu-2) \Gamma(\mu-1)} e^{-x} x^{\mu},
$$

in which $\Gamma(a)$ is the standard complete Gamma function. This stationary PDF depends only on the value of the $\beta$ parameter that controls the relative intensity of the multiplicative part of the perturbation and on the ratio $D / \lambda$ via the $\mu$ parameter of Equation (30). Notice that, as shown in Equation (24), $D / \lambda$ is the stationary variance of $T$. It is easy to check analytically that in the limit $\beta \rightarrow 0$ and $D / \lambda$ fixed, the stationary PDF in Equations (29)-(31) becomes a standard Gaussian, with the same variance 
$D / \lambda$. However, for $\beta \neq 0$, the stationary PDF is clearly non-Gaussian. In fact, for large positive $T$, it has a "power law" tail that makes it possible to have large fluctuations of positive values of $T$ (strong El Niño events). From Equation (30), we have that the $\mu$ parameter, which controls the behavior of this "heavy" tail of the stationary PDF, strongly depends on the value of the $\beta$ parameter. The maximum of the stationary PDF is found at $T_{\max }=-2 /(\beta \mu) \approx-2 \beta D / \lambda$; for fixed $D / \lambda$, it is proportional to $\beta$. The probability of strong negative values of $T$ (La Niña events) is largely reduced, and it is null for temperatures smaller than the threshold $T_{\min }=-1 / \beta$.

This skewed stationary PDF for $T$ fits well the data from observations (see Figure 1 ) with $\beta=0.2$ and $D / \lambda=0.8(\mu=32.7)$. The value for $D / \lambda$ is in agreement with the range of values we have already obtained for the variance of the ROM; hence, from now on, we set $D / \lambda=0.8$ and $\beta=0.2$.

From the FPE of Equation (17) and using the same ansatz that leads to the stationary PDF in Equations (29)-(31), it is also possible to obtain an analytical expression for the mean First Passage Time (FPT) for the ENSO events [77] and to compare these results with the observations. More precisely, we are interested in the average time we have to wait for the onset of a "strong" El Niño event, starting from a "neutral" initial condition defined as the case when the temperature $T_{i}$ has a value in the range $-0.5 \leq T_{i} \leq 0.5$. As depicted in Figure 6, "strong" El Niño events are identified by the criterion $T>1.5$. Thus, the FPT is defined as the time $\delta t\left(T_{i} \mid T_{t g}\right)$ when $T(t)$ first crosses a given target $T_{t g}$, starting from the initial value $T_{i}$ (see Figure 7). However, if $T(t)$ is a stochastic process, repeating many times the same "experiment" should lead to different values for $\delta t\left(T_{i} \mid T_{t g}\right)$, so that the FPT itself is a stochastic process, with its own PDF. We indicate with $t_{n}\left(T_{i} \mid T_{\operatorname{tg}}\right)$ the $n^{\text {th }}$ moment of the PDF of the FPT. Of course, the mean FPT is given by $t_{1}\left(T_{i} \mid T_{t g}\right)$. As we have shown in [77], we have:

$$
t_{1}\left(T_{i} \mid T_{t g}\right)=\left\{\frac{2}{p_{s}(T)} \frac{\beta}{2 \lambda} \frac{M\left[1,-(\mu-2),-\frac{\mu-2}{\beta T+1}\right]}{\beta T+1}\right\}_{T_{i}}^{T_{t g}}
$$

where $\{g(x)\}_{a}^{b} \equiv g(b)-g(a)$ and $M[1, \alpha, y]$ is the Kummers (generalized hypergeometric) function of the first kind with the first argument equal to one. We plot Equation (32) in Figure 8 to show the dependence of the mean FPT on $T_{t g}$ for different values of the $\lambda$ parameter.

From this figure, we see that $\lambda=1 / 12$ months $^{-1}$, i.e., a value at the lower end of the expected $\lambda$ range, provides the best agreement between observational estimates and our analytic result for the mean FPT. With $\lambda=1 / 12$ months $^{-1}$, the mean FPT for intermediate target temperatures, obtained from Equation (32), is in the range of 2-7 years, in good agreement with the observed intervals between intermediate El Niño events. Apart from the inverse proportionality relationship with the relaxation coefficient $\lambda$, it is also clear that the average FPT has a strong sensitivity to the value of the $\beta$ parameter (once the variance $\sigma^{2} \sim D / \lambda$ is kept fixed, the $\mu$ parameter depends only on $\beta$ as $\sim 1 / \beta^{2}$ ). In Figure 9, we compare the average FPT of Equation (32) with the values obtained through the numerical integration of the stochastic differential equation equivalent to the FPE of Equation (17) using $\beta=0.2$. We can see that there is a good agreement between the analytical and numerical solutions. In the same figure, we also show the analytical solutions of the average FPT in the case of a pure additive perturbation $(\beta=0$, in which case, the reduced stationary PDF for the $T$ variable is Gaussian) and for $\beta=0.3$. For weak to intermediate El Niño events $\left(T_{t g} \leq 1.5\right)$, the average FPT depends weakly on $\beta$. However, the sensitivity to $\beta$ clearly emerges in the case of strong and very strong events. In particular, the purely additive forcing leads to average FPT that are many orders of magnitude larger than those obtained with $\beta=0.2$ (note the logarithmic scale in the ordinate of the graph), while in the case of $\beta=0.3$, the FPTs are shorter at large $T_{t g}$. 
Notice that strong and very strong ENSO are extreme events, and as such, they are rare, so that it is not possible to have a sufficiently large observational sample size to validate in detail the analytical results. In fact, we do not use these extreme events to estimate the value of $\beta$ ( $\beta=0.2$ has been estimated by fitting to data the theoretical PDF of Equation (29), and it is compatible with both the observed negative value for the maximum of the PDF and its kurtosis). The other way around, we can use our analytical solution with $\beta=0.2$ as a reasonable extrapolation of the mean FPT for these strong or very strong El Niños, and the fact that this extrapolation fits well to the (few) available data is encouraging.

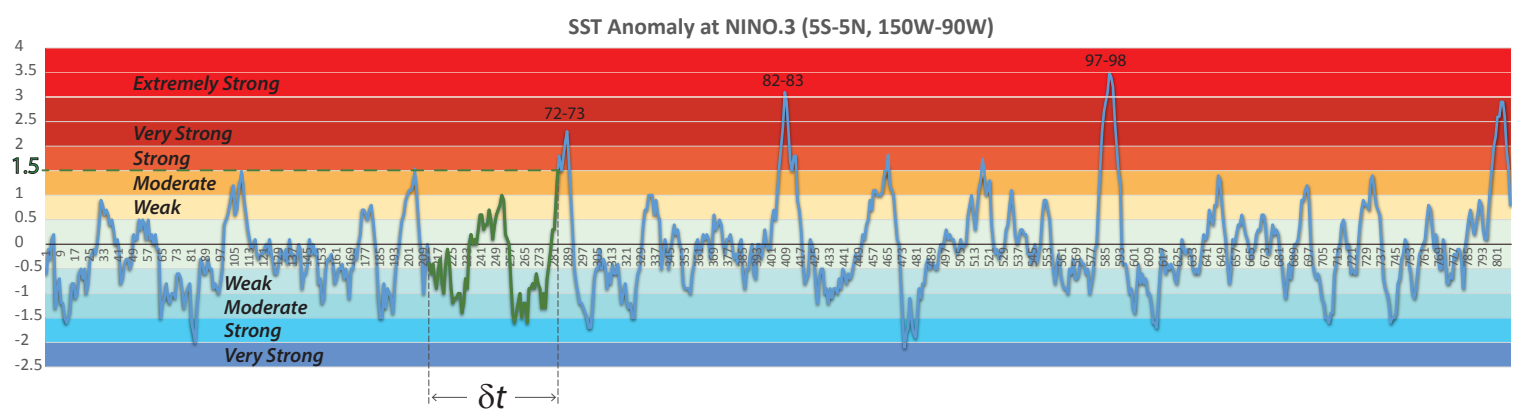

Figure 6. The month averaged Niño 3 data from January 1949-February 2016. Data are from the Tokyo Climate Center, WMO Regional Climate Centers (RCCs) [78]. In green, we highlight a segment of the time series to illustrate the First Passage Time (FPT) $\delta t\left(T_{i}, T_{t g}\right)$ for a given target temperature anomaly $T_{t g}$ (here, $\left.T_{t g}=1.5\right)$, starting from an initial neutral temperature $T_{i}\left(-0.5 \leq T_{i} \leq 0.5\right)$. See also the text and Figure 7 for details about the FPT.

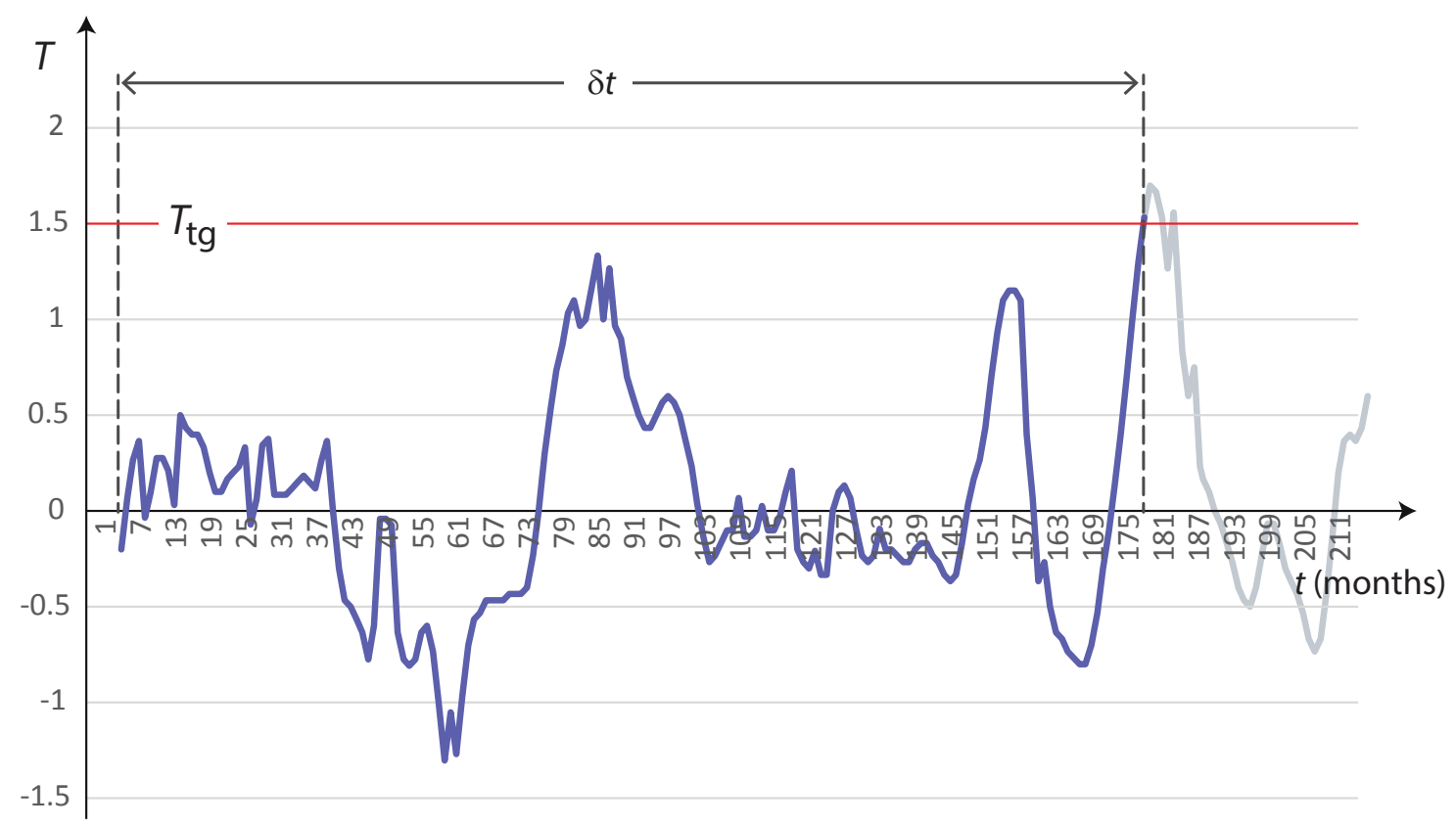

Figure 7. The first passage time $\delta t\left(T_{i}, T_{t g}\right)$ for a target temperature anomaly of $T_{t g}=1.5$ is defined as the first time the fluctuating temperature $T(t)$ crosses the threshold $T_{t g}$ (1.5 in this case), starting from an initial temperature $T_{i}$ (that we will choose in the range $-0.5 \leq T_{i} \leq 0.5$, corresponding to neutral ENSO conditions). 


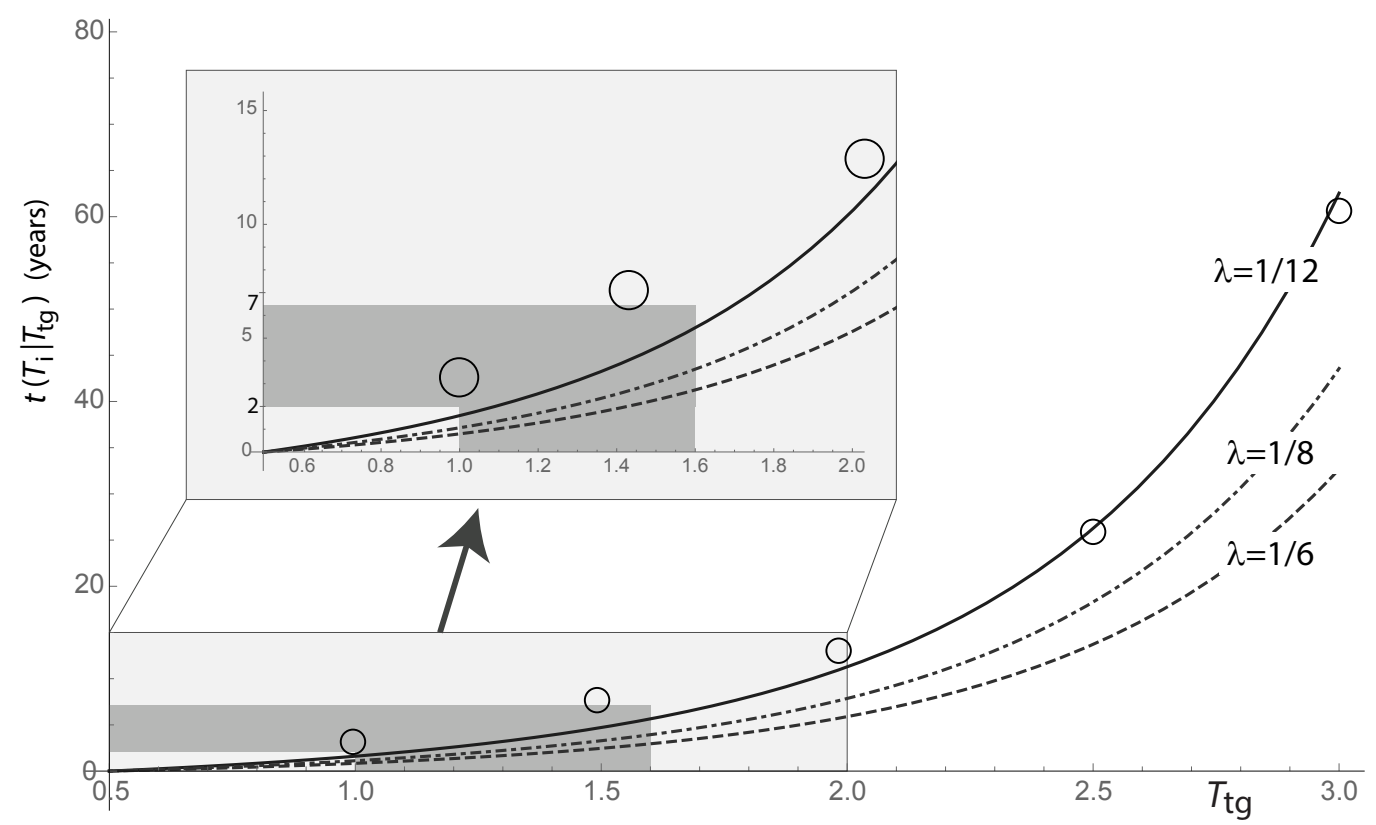

Figure 8. The mean FPT for different values of the $\lambda$ parameter, vs. the target temperature, obtained using Equation (32), compared with observations (circles). The values of $\beta$ and $\mu$ have been fixed by fitting the stationary PDF of Equations (29)-(31) to Niño 3 from [50]: $\beta=0.2$ and $\mu=32.7$ (see the text for details). Dashed line: $\lambda=1 / 6$ month $^{-1}$, dotted-dashed line $\lambda=1 / 8$ month $^{-1}$, solid line $\lambda=1 / 12$ month $^{-1}$. In the inset, a zoom of the same graphs, where the gray background emphasizes the range of $2-7$ years and $1.0 \leq T \leq 1.6$, corresponding to the typical recurring time interval for intermediate El Niño events. Notice how the curve obtained with $\lambda=1 / 12$ month $^{-1}$ falls better than the others in this zone.

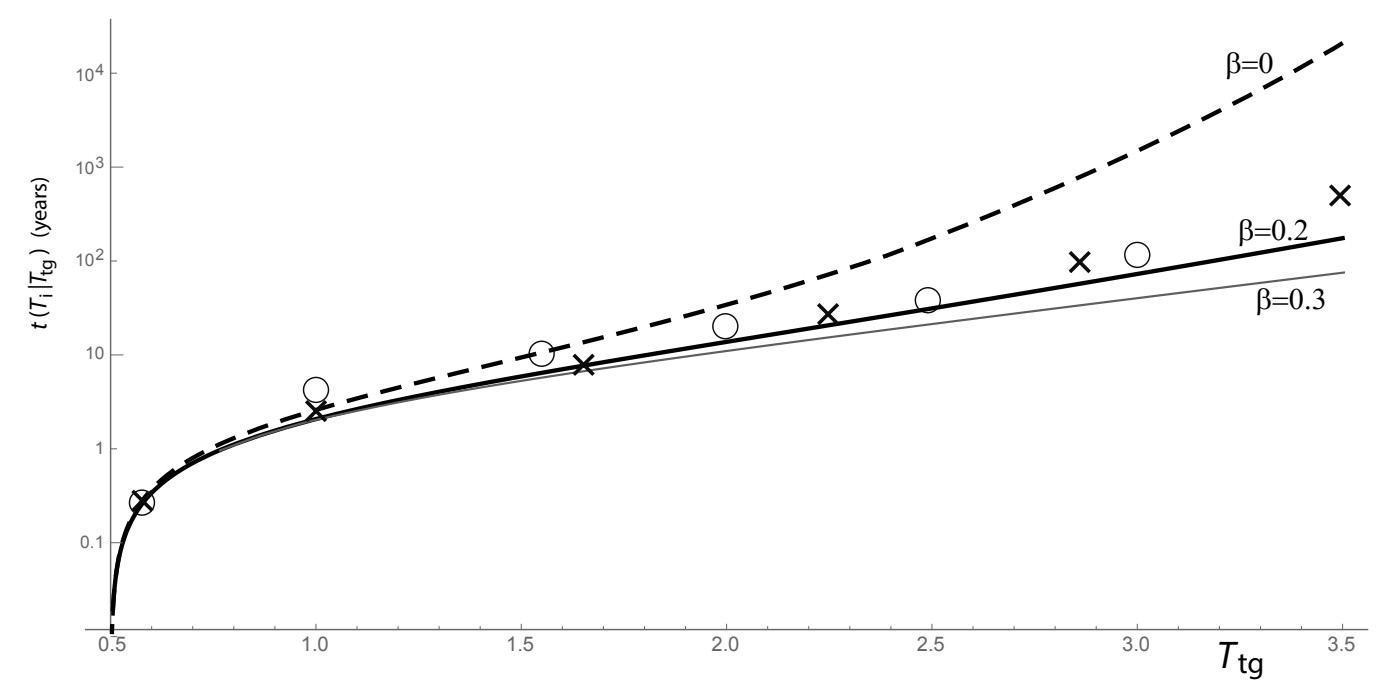

Figure 9. Semi-log plot of the average FPT as a function of the target temperature $T_{t g}$, for $\lambda=$ $1 / 12$ month $^{-1}$ and $\sigma^{2}=D / \lambda=0.8$ (see the text for details). Thick and thin solid lines show analytical solutions from Equation (32) with $\beta=0.2$ and $\beta=0.3$, respectively; while the thick dashed line is for the case $\beta=0$, corresponding to the pure additive forcing of the ROM (in this case, the stationary PDF is the Gaussian). Circles: the average FPT from Niño 3 data from NOAA [50]. Crosses: average FPT from numerical simulations of the Îto SDE corresponding to the FPE of Equation (17) [79]: $\mathrm{d} h=-\omega T \mathrm{~d} t$; $\mathrm{d} T=(-\omega h+D \beta) \mathrm{d} t-\left(\lambda-D \beta^{2}\right) T \mathrm{~d} t+\sqrt{D}(1+\beta T), \mathrm{d} W$, where $W$ is a Wiener process, $\beta=0.2$, and $\omega=2 \pi / 48$ month $^{-1}$. 


\subsection{Inferring the FPE Coefficients from Data}

The results of the previous sections validate the FPE of Equation (17) with parameters $\omega=2 \pi$ $/ 48$ month $^{-1}, \lambda=1 / 12$ month $^{-1}$ and $D / \lambda=0.8$. However, at least in principle, there is a way to infer the transport coefficients of an FPE directly from data. For that purpose, let us rewrite the FPE of Equation (17) in the following way:

$$
\partial_{t} \sigma(h, T ; t)=\left\{-\partial_{h} G_{h}(h, T)-\partial_{T} G_{T}(h, t)+\partial_{T}^{2} A(T)\right\} \sigma(h, T ; t),
$$

where (as in Equation (23) we used the transformation $h \rightarrow h+\beta D / \omega$ to eliminate the mean value of the thermocline depth anomaly):

$$
\begin{aligned}
A(T) & \equiv D(1+\beta T)^{2} \\
G_{T}(h, T) & \equiv \omega h-\left(\lambda-D \beta^{2}\right) T \\
G_{h}(h, T) & \equiv-\omega T .
\end{aligned}
$$

It is well known that given the FPE of Equation (17), the drift and diffusion coefficients can be inferred using their statistical definitions as conditional first and second moments of the process increments [80-82]:

$$
\begin{aligned}
A(T) & =\frac{1}{2} \lim _{\delta t \rightarrow 0} \frac{1}{\delta t} \int_{-\infty}^{\infty}\left(T-T^{\prime}\right)^{2} P\left(h^{\prime}, T^{\prime} \mid h, T ; \delta t\right) \mathrm{d} h^{\prime} \mathrm{d} T^{\prime} \\
G_{T}(h, T) & =\lim _{\delta t \rightarrow 0} \frac{1}{\delta t} \int_{-\infty}^{\infty}\left(T-T^{\prime}\right) P\left(h^{\prime}, T^{\prime} \mid h, T ; \delta t\right) \mathrm{d} h^{\prime} \mathrm{d} T^{\prime} \\
G_{h}(h, T) & =\lim _{\delta t \rightarrow 0} \frac{1}{\delta t} \int_{-\infty}^{\infty}\left(h-h^{\prime}\right) P\left(h^{\prime}, T^{\prime} \mid h, T ; \delta t\right) \mathrm{d} h^{\prime} \mathrm{d} T^{\prime},
\end{aligned}
$$

in which $P\left(h^{\prime}, T^{\prime} \mid h, T ; \delta t\right)$ is the conditional probability to go from $(h, T)$ to $\left(h^{\prime}, T^{\prime}\right)$ in an infinitesimal time $\delta t$. From a practical viewpoint, the above expressions are obtained from the expectation values of the first (Equations (35b) and (35c)) and second (Equation (35a)) moments of the difference of two subsequent Niño 3 values, where the time step is $\delta t=1$ month. Because the duration of the $h$ time series is too short to obtain a reliable two-dimensional PDF (note that for $h$, we use the "proxy" given by the anomalies in the volume of Warm Water (WWV) in the basin $120^{\circ} \mathrm{E}-80^{\circ} \mathrm{W}, 5^{\circ} \mathrm{S}-5^{\circ} \mathrm{N}$, in the time range from January 1982-December 2017, taken from [76]), we focus our attention only on the $T$ variable, i.e., we work only with the observed Niño 3 index. This means that we disregard the drift coefficient of Equation (35c), and for each $T$ value, when we evaluate the expectation of the first and second moments of the process increments of $T$, we automatically average over $h$ at fixed $T$. This average over the $h$ variable does not affect the diffusion coefficient of Equation (35a), thus,

$$
\frac{\mathbb{E}\left[(T(t+\delta t)-T(t))^{2}\right]}{2 \delta t}=A(T)=D(1+\beta T)^{2},
$$

while for the drift coefficient of Equation (35b), we have:

$$
\begin{aligned}
\frac{\mathbb{E}[T(t+\delta t)-T(t)]}{\delta t}=\left\langle G_{T}(h, T)\right\rangle_{T, S} & \equiv \omega\langle h\rangle_{T, S}-\lambda T+\alpha \epsilon_{1}^{2} \beta(1+\beta T) \\
& \sim-\left(\lambda-D \beta^{2}\right) T \simeq-\lambda T
\end{aligned}
$$

where $\langle\ldots\rangle_{T, s}$ means the conditional stationary average at fixed $T$, and in the second line, we have exploited the ansatz introduced in [17] (see also Appendix C). 
The functions in Equations (36) and (37)) correspond to the diffusion and drift coefficients, respectively, of the following reduced FPE for the $T$ variable:

$$
\partial_{t} p(T ; t)=\left\{\left(\lambda-D \beta^{2}\right) \partial_{T} T+\partial_{T}^{2} D(1+\beta T)^{2}\right\} p(T ; t),
$$

that is, the same FPE we would obtain directly by using the already cited ansatz introduced in [17], in the FPE of Equations (17)-(34). In Figure 10, we see that from the Niño 3 data, the expectation value, $\mathbb{E}[T(t+\delta t)-T(t)]$ looks as a linear function of $T$, supporting again the view of a linear unperturbed ROM, and the linear fit is very close to $-\lambda T$ with $\lambda=1 / 12$ month $^{-1}$, the same values we have obtained from the mean FPT analysis of the previous subsection.

Concerning the diffusion coefficient, evaluated as in Equation (35a), we see from Figure 11 that the expectation value $\mathbb{E}\left[(T(t+\delta t)-T(t))^{2}\right] / 2$ is compatible with the square dependence on $T$ of Equation (36), but the the uncertainty is large. This large uncertainty is due to the nonlinear nature of $A(T)$, which makes the fitting process strongly dependent on large $T$ values where the statistics is poor. The large value of the $\mathbb{E}\left[(T(t+\delta t)-T(t))^{2}\right] / 2$ for large negative $T(\sim-2)$ suggests that $\beta$ could be quite larger than 0.2 (leading to a diffusion term more symmetric with respect to zero compared with the case $\beta=0.2$ ); however, in this case, it would be necessary to add another noise term, additive and uncorrelated with the CAM one,to explain the other results so far presented. Actually, this is the perturbation already introduced in Equation (16), and the corresponding diffusion coefficient is shown in the same Figure 11 as a dashed green line. The case where an additional uncorrelated noise is added to the present perturbed ROM will be deeply analyzed in a forthcoming work.

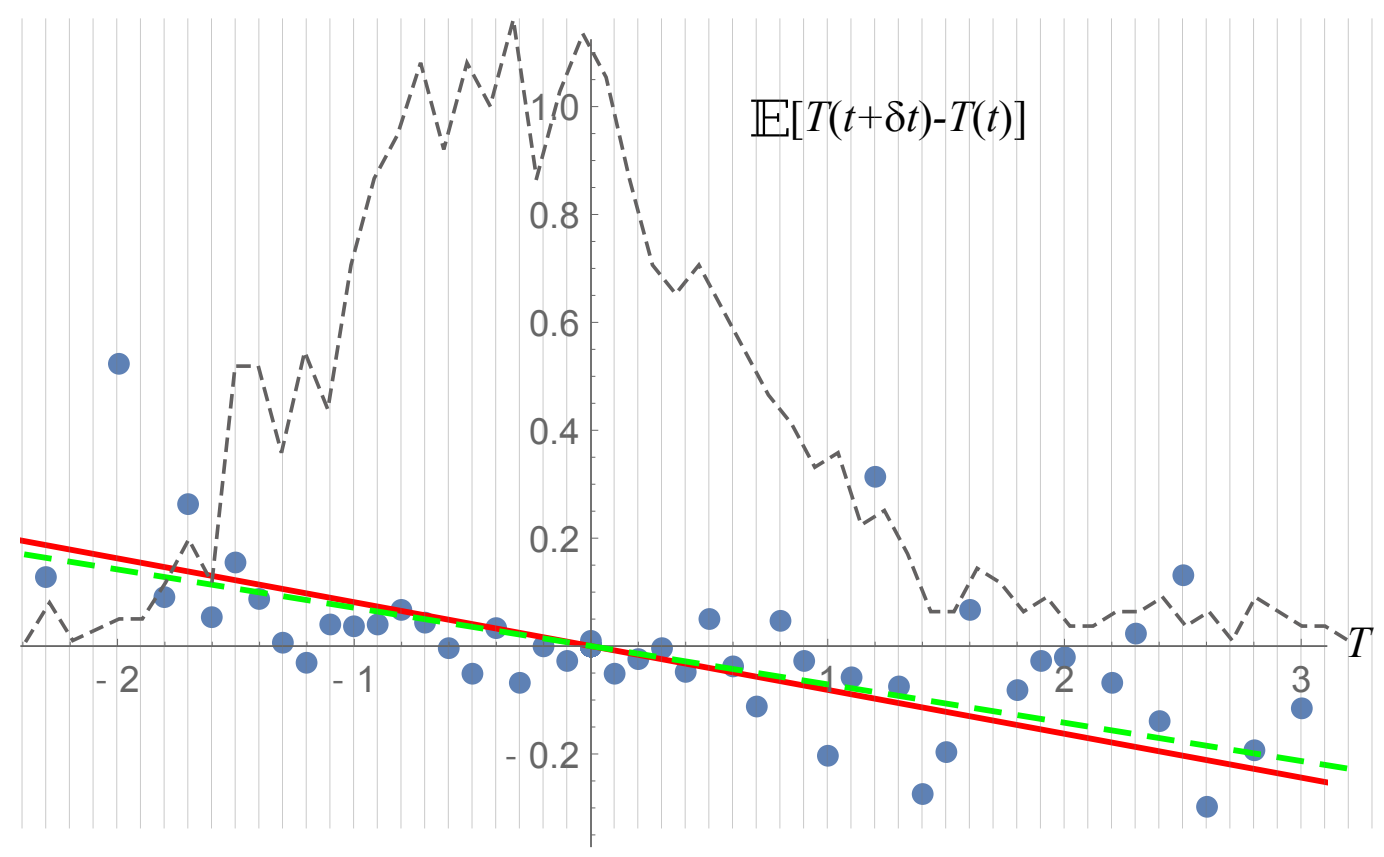

Figure 10. Dots: the expectation value $\mathbb{E}[T(t+\delta t)-T(t)]$ where $\delta t=1$ month, of the Niño 3 data from NOAA [50]compared with the drift coefficient $\left\langle G_{T}(h, T)\right\rangle_{T, s} \simeq-\lambda T$ (see Equation (37)), in the cases where the coefficient $\lambda$ is obtained as the the best fit to the data (dashed green line), from which $\lambda=1 / 13.7$ month $^{-1}$, close to the case where $\lambda=1 / 12$ month $^{-1}$ (solid red line) that corresponds to the value obtained fitting the correlation functions and the average timing of the ENSO events (see the text for details). Gray dashed line: number of observed events for each value of $T$, scaled by a factor $1 / 76$ in the vertical axes. For large absolute values of $T$, the spreading of the data around the linear fit increases because the number of registered ENSO events decreases (e.g., just one for $T=-2$ ). 


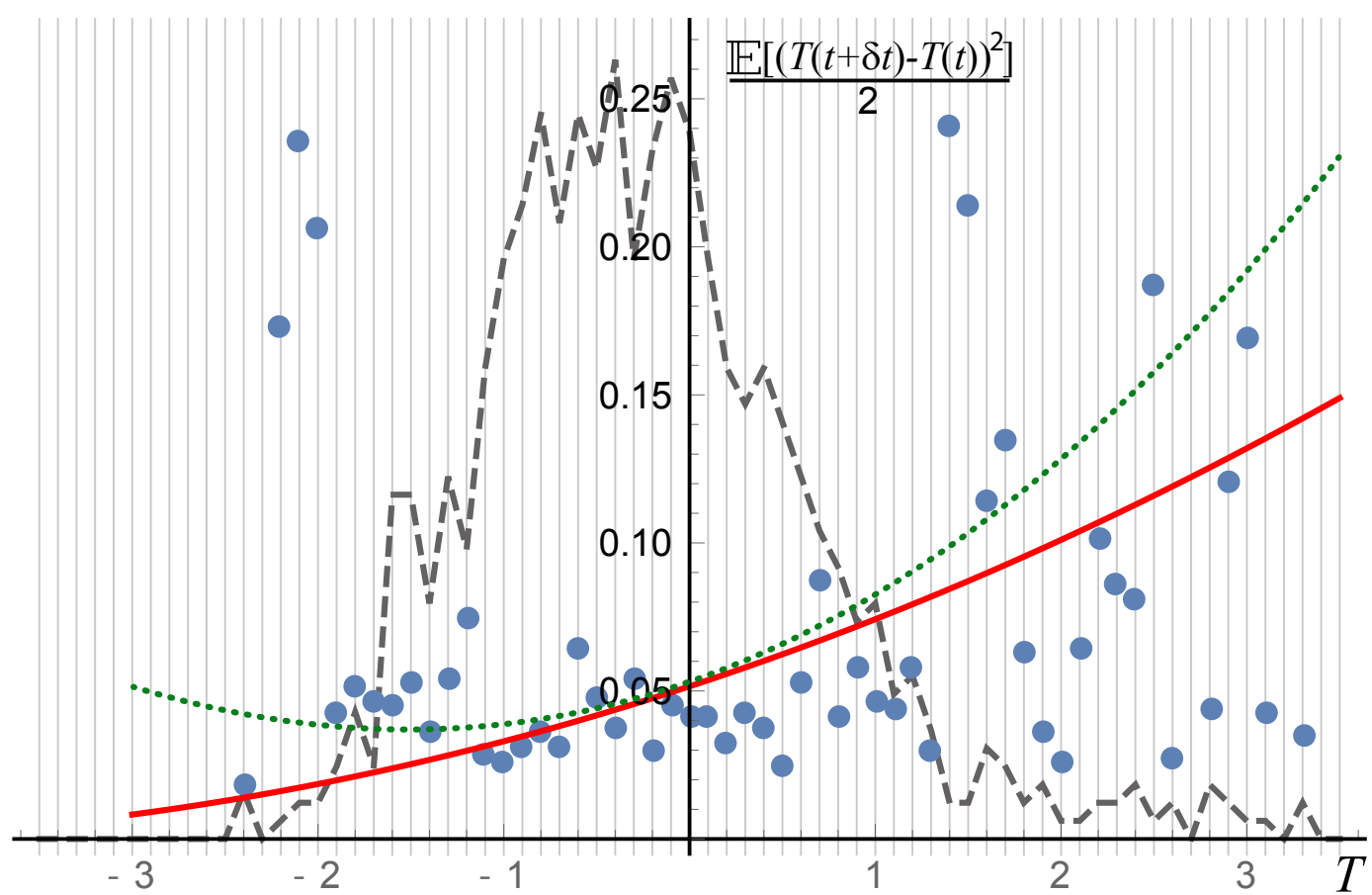

Figure 11. Dots: the expectation value $\mathbb{E}\left[(T(t+\delta t)-T(t))^{2}\right] / 2$ where $\delta t=1$ month, of the Niño 3 data from NOAA [50]. Solid red line: diffusion term $A(T)=D(1+\beta T)^{2}$ (see Equation (36)), where $\beta=0.2$ and $D=\sigma^{2} \lambda=0.07$ month $^{-1}$ (see the text for details). Gray dashed line: the number of observed events for each value of $T$, scaled by a factor $1 / 160$ in the vertical axes. The points for extreme events are not that close to the red line, but in these cases, the statistics is also really poor. Green dotted line, function $D(1+\beta T)^{2}+D_{1}$, with the following figures obtained from the fit to the data with fit parameters $\beta$ and $D\left(D_{1}=\sigma^{2} \lambda-D\right.$ to ensure a fixed variance of the PDF): $\beta=0.9, D=0.011$ month $^{-1}$ and $D_{1}=\sigma^{2} \lambda-D=0.07$ month $^{-1}-0.011$ month $^{-1}=0.059$ month $^{-1}$.

\section{Discussion and Conclusions}

Starting from the fact that the ENSO statistics has some clear non- Gaussian features, in this paper, we use the results of some recent papers, and we analyze further the statistics of the ENSO, with the specific goal of discussing which non-linear dynamics might be responsible for them. Thus, as in [17], we work with the Fokker-Planck Equation (FPE) for the PDFof the ROM variables, but here aiming at the search for nonlinearities. In this simplified model, we consider two possible sources of nonlinearities: nonlinearity in the dynamics of the unperturbed ROM and a nonlinear (i.e., multiplicative) fast perturbation of the ROM.

Comparison with observations leads to the conclusion that a possibly nonlinear interaction between the internal ROM variables cannot be inherently the cause of the observed non-Gaussianity, which instead is well reproduced assuming a linear ROM with a nonlinear chaotic perturbation.

The hypothesis of nonlinear interaction between the unperturbed ROM and the fast forcing is strongly supported by the comparison between observations and theoretical results (stationary PDF and average FPT) for values of $T$ where the number of observation data is statistically relevant (weak and intermediate ENSO).

It is noticeable that extrapolating this result also for large $T$, the heavy tail of the PDF leads to relatively short recurring times of strong ENSO, which still fit well to the few observations and that are orders of magnitude smaller than those we would have in the Gaussian case.

In Section 6.4 for the first time, observation data are used to infer directly the FPE coefficients. In this way, we are able to validate once more the assumption of a linear internal dynamics of the ROM (Figure 10) and of a non-constant diffusion (Figure 11) coefficient. At a quantitative level, however, the exact relationship between $T$ and the diffusion coefficient is difficult to determine in this way 
because nonlinearities become important in the large $T$ range (rare events), where the statistics is rather poor (dashed line in Figure 11).

We can conclude that the nonlinear interaction between the fast and slow modes of the ENSO gives a substantial contribution to the non-Gaussianity of the ENSO statistics. However, further work is necessary to better quantify the nonlinear nature of the perturbation, which is related to the nonlinear diffusion coefficient of the FPE. For example, from observations, we see that the expectation value $\mathbb{E}\left[(T(t+\delta t)-T(t))^{2}\right] / 2$, which should reproduce the functional dependence of the diffusion coefficient with respect to the $T$ variable, is compatible with the CAM diffusion term $A(T)=D(1+$ $\beta T)^{2}$ of Equation (36) with a small beta $(\beta \sim 0.2)$, but also with the case where there is another diffusion coefficient $D_{1}$, in this case constant. In fact, it turns out that the sum $D_{1}+A(T)$ fits to the data, as well as $A(T)$ alone (see in Figure 11), but with an increased value for $\beta$ and a decreased value for $D$ (the sum $D+D_{1}$ must be kept fixed to preserve the value of the variance). Thus, to be able to define the actual $D_{1}$ and $\beta$ values, we should increase the sample size used for the fitting process, for example using longer time series from century-long reanalysis products (CERA 20C, https:// www.ecmwf.int/en/forecasts/datasets/archive-datasets/reanalysis-datasets/cera-20c) and/or proxy data (networks of precipitation, tree-rings, corals and ice core records) that can help extend the time series back in time some hundreds of years [83]. Another point that deserves more investigation is the role of the weak nonlinearity in the interaction between the ROM variables $h$ and $T$, under the current assumption of the nonlinear perturbation. In fact, in Section 3, we have assumed a linear (i.e., additive) perturbation to show that the internal nonlinearities cannot be the cause of the observed non-Gaussianity of the Niño 3 PDF. However, how much does the internal nonlinearity affect the PDF in the case of a nonlinear interaction? This is not a trivial question because, as we have shown in this paper, the nonlinear interaction leads to a much slower decay of the PDF, with respect to the linear (i.e., additive) case, and the tails of the PDF are where the small nonlinear terms can emerge. These are all issues on which we are currently working.

Author Contributions: Conceptualization, M.B. Formal analysis, M.B., R.M. and S.M. Data, M.B., R.M., S.M. and A.C. Methodology, M.B., R.M. and A.C. Validation, R.M. and A.C. Writing-original draft, M.B. and S.M. Writing-review and editing, M.B., A.C., R.M. and S.M.

Funding: This research received no external funding.

Conflicts of Interest: The authors declare no conflict of interest.

\section{Appendix A. Validation of the Linear Relationship between the Wind Stress and $T_{E}$}

Equation (4) is an assumption that agrees with observations, as shown in the following. The wind stress is considered proportional to the square of the wind velocity at $10 \mathrm{~m}$ from the sea surface, taken from [61]. Figure A1 shows the equatorial zonal (i.e., averaged over $5^{\circ} \mathrm{S}-5^{\circ} \mathrm{N}$ ) wind velocity for different longitudes. We see that very close to the Americas' coasts $\left(81^{\circ} \mathrm{W}\right)$, the direction of the wind flips from trade wind to westerly. This is due to the thermal gradient we have when we pass from the cold East Pacific Equatorial Ocean to the warm Central American lands. Thus, for the wind stress $\tau_{s}$ of Equation (4), we use the anomaly (i.e., 1948-2018 mean removed) of the equatorial zonal wind stress averaged in the longitude interval $180^{\circ} \mathrm{W}-120^{\circ} \mathrm{W}$. The fluctuating quantity $\tau_{s}$ is divided into slow $\left(\tau_{s, \text { slow }}\right)$ and fast $\left(\tau_{s, \text { fast }}\right)$ components. The $\tau_{s, \text { slow }}$ data are obtained from the one-year average of $\tau_{s}$ data, while $\tau_{s, \text { fast }} \equiv \tau_{s}-\tau_{s, \text { slow }}$. Then:

(1) From Figure A2, we clearly see that the time behaviors of $\tau_{s, \text { slow }}$ and $T_{E, \text { slow }}$ are similar (of course, some lag remains between the wind stress, which is a a forcing term, and the ocean reaction);

(2) From Figure A3, we see that the plot of the cross-correlation between $\tau_{s, \text { slow }}$ and $T_{E, \text { slow }}$ vs. time (in moths) is very similar to the plot of the autocorrelation of $T_{E, \text { slow }}$ (notice the little time offset between these two plots, indicating the fact that the one-year time average has not completely hidden the cause-effect relationship between $\tau_{s, \text { slow }}$ and $\left.T_{E, \text { slow }}\right)$. Moreover, from the insert of the same figure, we see that the average relationship between $\tau_{s, \text { slow }}$ and $T_{E, \text { slow }}$ is mainly linear, with 
a small dispersion of the data around the linear fit (quantities are normalized by the standard deviation of the quantities from observations):

$$
\tau_{s} \equiv \tau_{s, \text { slow }}+\tau_{s, f a s t}=k T_{E}+\tau_{s, f a s t}
$$

The above two arguments strongly support the hypothesis expressed by Equation (4).

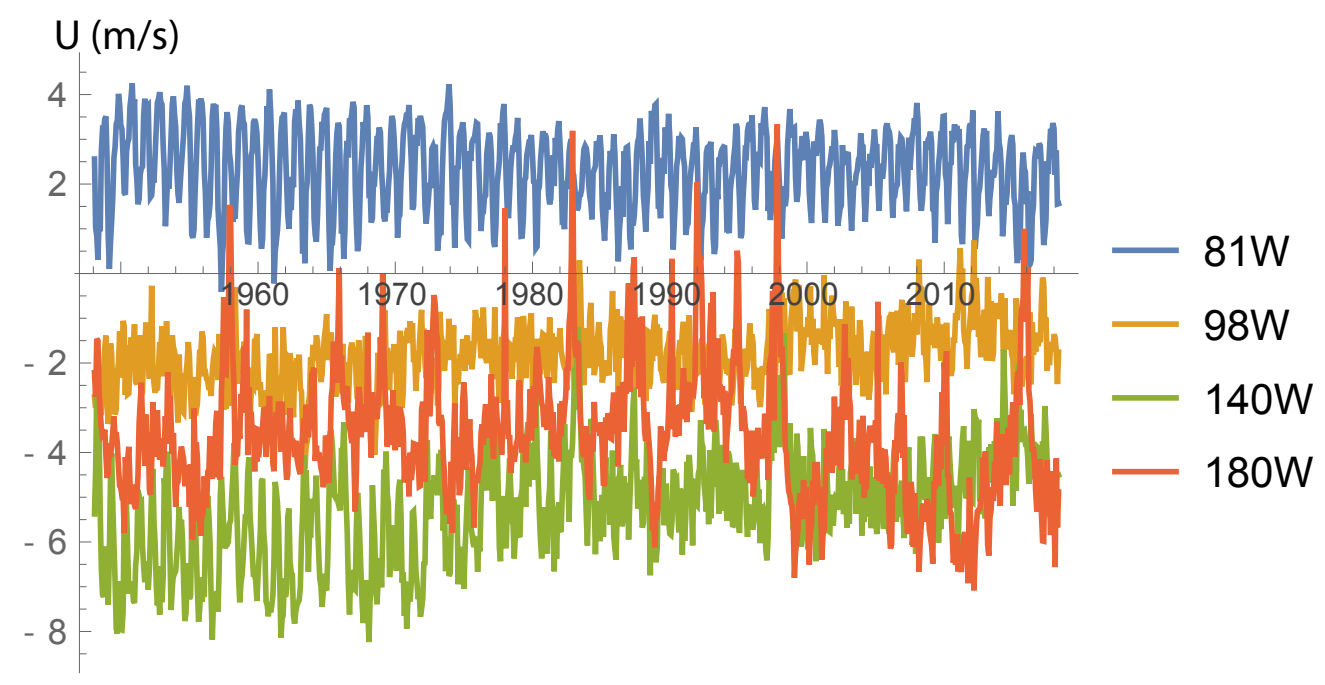

Figure A1. 5N-5S averaged zonal wind for different longitudes. Data from NOAA [61].

\section{Appendix B. Very Short Review of the Projection Approach Applied to the ROM}

In the present work, we study the same ROM considered in [17]; however, we do not use the FPE in Equations (3) and (4) of [17] because of a marginal mistake: a missing additive term in the FPE, which here we want to fix. Thus, for the reader's convenience, in the following, we give a short summary of the projection procedure adapted to the present case.

The idea of this approach is to assume that the fast fluctuating force $\xi(t)$ has a deterministic nature, namely that the ROM of Equation (15) is just a part of a set of equations describing a larger deterministic system:

$$
\begin{aligned}
\dot{h} & =-\omega T \\
\dot{T} & =\omega h-\lambda T+\epsilon \xi(1+\beta T) \\
\dot{\xi} & =F(\xi, \pi) \\
\dot{\pi} & =Q(\xi, \pi) .
\end{aligned}
$$

Using the terminology of the projection approach [27,66,84], the ROM is viewed as the "system of interest" (or system $a$ ), while $(\xi, \pi)$ represents the booster system (or "rest of the system" or system $b$ ), namely a set of general chaotic and fast variables, e.g., the MJO and WWB [85-89], which, perturbing the ROM, activate the El Niño/La Niña phenomena and which obey some unspecified equations of motion expressed by the generic functions $F(\xi, \pi)$ and $Q(\xi, \pi)$. The value of the parameter $\epsilon$ determines the intensity of the perturbation to the ROM. For $\epsilon=0$, the first two lines of Equation (A2) define the unperturbed system of interest (or unperturbed ROM).

We stress that we leave unspecified the exact expressions of the functions $F(\xi, \pi)$ and $Q(\xi, \pi)$, representing the equation of motion for the booster, because we do not need to know them in detail (the booster typically will be a nonlinear chaotic system); it is enough that they satisfy some specific assumptions, as detailed in the following. 
More generally, the interaction between the ROM and the booster should be bi-directional: the booster equation of motion should be affected by the dynamics of the ROM: $F=F(\xi, \pi, \epsilon R(h, T))$ and $Q=Q(\xi, \pi, \epsilon R(h, T))$. The function $\epsilon R(h, T)$ is the "reaction" force of the ROM variables on the $\mathrm{MJO} / \mathrm{WWB}$ system. However, for the sake of simplicity, we shall consider hereafter $\epsilon R(h, T)=0$ as in [17]. The feedback term can be included following the general approach of [26,33]. The goal is to describe the statistics of the part of interest. We start from the following Langevin equation for the $\operatorname{PDF} \rho(h, T, \xi, \pi ; t)$ of the total system:

$$
\partial_{t} \rho(h, T, \xi, \pi ; t)=\left\{\mathcal{L}_{a}+\epsilon \mathcal{L}_{I} \xi+\mathcal{L}_{b}\right\} \rho(h, T, \xi, \pi ; t)
$$

where the unperturbed and perturbation Liouville operators are given by:

$$
\begin{aligned}
& \mathcal{L}_{a}=\omega \partial_{h} T-\omega \partial_{T} h+\lambda \partial_{T} T \\
& \mathcal{L}_{I}=-\partial_{T}(1+\beta T),
\end{aligned}
$$

respectively. In Equation (A3), we do not write the explicit expression of the Liouville operator $\mathcal{L}_{b}$ of the booster because it is related to the unknown functions $F(\xi, \pi)$ and $Q(\xi, \pi)$.

We are interested in obtaining a Fokker-Plank Equation (FPE) for the reduced (or marginal) PDF of the system of interest, given by $\sigma(h, T ; t) \equiv \int \rho(h, T, \xi, \pi ; t) \mathrm{d} \xi \mathbf{d} \pi$. Introducing the projection operator, $\mathbb{P} \cdots \equiv \wp_{b}(\xi, \pi) \int \mathrm{d} \xi \mathbf{d} \pi \cdots$, where $\wp_{b}$ is the stationary PDF of the booster, defined by $\wp_{b}(\xi, \pi) \mid \mathcal{L}_{b} \wp_{b}(\xi, \pi)=0$, we have: $\sigma(h, T ; t)=1 / \wp_{b}(\xi, \pi) \times \mathbb{P} \rho(h, T, \xi, \pi ; t)$. Following the Zwanzig-like formal projection approach in the perturbation version of [64-66] to the lowest non-vanishing order on the coupling parameter $\epsilon$, the time evolution of $\sigma(h, T ; t)$ is governed by the following integro-differential equation:

$$
\begin{aligned}
& \partial_{t} \sigma(h, T ; t)=\mathcal{L}_{a} \sigma(x ; t) \\
& +\epsilon^{2}\left\langle\xi^{2}\right\rangle_{b}\left\{\mathcal{L}_{I} \int_{0}^{\infty} \mathrm{d} u \varphi(u) e^{\mathcal{L}_{a} u} \mathcal{L}_{I} e^{-\mathcal{L}_{a} u}\right\} \sigma(h, T ; t)
\end{aligned}
$$

In the above equation, $\varphi(u)$ is the normalized auto-correlation function of the booster variable $\xi$ and $\left\langle\xi^{2}\right\rangle_{b}$ is the variance of $\xi$ (without any loss of generality, we assumed $\langle\xi\rangle_{b}=0$ ). A very important outcome of Equation (A5) is that we can group all the possible booster dynamical systems in different classes of equivalence, where all the boosters belonging to the same class give rise to the same statistical properties for a given system of interest. In fact, the FPE depends only on the booster autocorrelation function $\left\langle\xi^{2}\right\rangle_{b} \varphi(u)$ : different dynamical systems that share the same autocorrelation function belong to the same booster class of equivalence. Given Equation (A4), we can rewrite Equation (A5) as:

$$
\begin{aligned}
& \partial_{t} \sigma(h, T ; t)=\left\{\partial_{h} \omega T-\partial_{T} \omega h+\lambda \partial_{T} T\right. \\
& +\beta \epsilon^{2}\left\langle\xi^{2}\right\rangle_{b} \tau \partial_{T}(1+\beta T) \\
& +\epsilon^{2}\left\langle\tilde{\zeta}^{2}\right\rangle_{b} \partial_{T}(1+\beta T) \\
& \left.\times \int_{0}^{\infty} \mathrm{d} u \varphi(u)\left(1+\beta T_{a}(h, t ;-u)\right) e^{\mathcal{L}_{a} u} \partial_{T} e^{-\mathcal{L}_{a} u}\right\} \sigma(h, T ; t),
\end{aligned}
$$

where we have used the identity $\partial_{T}(1+\beta T)=(1+\beta T) \partial_{T}+\beta$ (this last term was the cited missed one in [17], and it is not vanishing when the interaction with the booster is not Hamiltonian, as in the present case), and we have introduced the decay time of the booster autocorrelation function as $\tau \equiv \int_{0}^{\infty} \mathrm{d} u \varphi(u)$. Moreover, in the above equation, we have exploited the result $\exp \left[\mathcal{L}_{a} u\right] T \exp \left[-\mathcal{L}_{a} u\right]=T_{a}(h, t ;-u)$ (see for example Equations (31) and (32) of [27]), where $T_{a}(h, t ;-u)$ and $h_{a}(h, t ;-u)$ (for further use) are the unperturbed $(\epsilon=0)$ back time evolution for a time $u$ of the $T$ and the $h$ variables, respectively, starting from the initial condition $(u=0)$ given by $T_{a}(h, t ; 0)=T$ and $h_{a}(h, t ; 0)=h$. 
In the case where there is an extremely large time scale separation between the dynamics of the unperturbed ROM and the forcing atmosphere, the autocorrelation function $\varphi(u)$ decays so fast, with respect to the typical time scale of the unperturbed ROM, that we can make the following approximations: $T_{a}(h, t ;-u) \sim T$ and $e^{\mathcal{L}_{a} u} \partial_{T} e^{-\mathcal{L}_{a} u} \sim \partial_{T}$. Thus, in this case, from Equation (A6), we obtain the standard result of the theory of stochastic Markovian processes [74], namely the FPE of Equation (17).

More in general, Equation (A6) gives the transport coefficients $A(h, T)$ and $B(h, T)$ of Equation (18), also for the cases where we do not assume such stringent assumptions about the time scale separation between the unperturbed ROM and the fast atmosphere. These have been already given in the Supporting Information of [17], by making a link with some final results of [27]. We mention only the fact that the most troublesome problem in order to get $A(h, T)$ and $B(h, T)$ from Equation (A6) is to manipulate the compound of operators $\exp \left[\mathcal{L}_{a} u\right] \partial_{T} \exp \left[-\mathcal{L}_{a} u\right]$. For that, we refer the reader to the results of [33] and (for a more general approach to the Lie evolution of differential operators) to the more recent work of [32]. Here, we report the final result, which is given by Equation (19), where $\left(D \equiv \epsilon^{2}\left\langle\tilde{\xi}^{2}\right\rangle_{b} \tau\right)$ :

$$
\begin{aligned}
& A_{0}=\frac{D}{\tau} \int_{0}^{\infty} d u \varphi(u) e^{-\frac{\lambda}{2} u}\left[\cos (\Omega u)-\frac{\lambda}{2 \Omega} \sin (\Omega u)\right] \\
& =\frac{D}{\tau}\left\{\Re[\hat{\varphi}(\Omega-\mathrm{i} \lambda / 2)]+\frac{\lambda}{2 \Omega} \Im[\hat{\varphi}(\Omega-\mathrm{i} \lambda / 2)]\right\} \\
& A_{1}=-\frac{D}{\tau} \frac{\omega}{2 \Omega} \int_{0}^{\infty} d u \varphi(u)\left[\frac{\lambda}{2 \Omega} \cos (2 \Omega u)+\sin (2 \Omega u)\right] \\
& +\frac{D}{\tau} \frac{\omega \lambda}{4 \Omega^{2}} \int_{0}^{\infty} \varphi(u) d u \\
& =-\frac{D}{\tau} \frac{\omega}{2 \Omega}\left\{\frac{\lambda}{2 \Omega} \Re[\hat{\varphi}(2 \Omega)]-\Im[\hat{\varphi}(2 \Omega)]\right\} \\
& +D \frac{\omega \lambda}{4 \Omega^{2}} \\
& \begin{aligned}
A_{4} & =\frac{D}{\tau} \int_{0}^{\infty} d u \varphi(u)\left[\omega^{2}\left(1+\cos (2 \Omega u)-\frac{\lambda^{2}}{2}\right] \frac{1}{2 \Omega^{2}}\right. \\
& =D\left(1-\frac{\omega^{2}}{2 \Omega^{2}}\right)+\frac{D}{\tau} \frac{\omega^{2}}{2 \Omega^{2}} \Re[\hat{\varphi}(2 \Omega)]
\end{aligned} \\
& A_{2}=A_{4}+A_{0}, \quad A_{3}=A_{1} \\
& B_{0}=-\frac{D}{\tau} \frac{\omega}{\Omega} \int_{0}^{\infty} d u \varphi(u) e^{-\frac{\lambda}{2} u} \sin (\Omega u) \\
& =\frac{D}{\tau} \frac{\omega}{\Omega} \Im[\hat{\varphi}(\Omega-i \lambda / 2)] \\
& B_{1}=-\frac{D}{\tau} \frac{\omega^{2}}{2 \Omega^{2}} \int_{0}^{\infty} d u \varphi(u) \cos (2 \Omega u) \\
& +\frac{D}{\tau} \frac{\omega^{2}}{2 \Omega^{2}} \int_{0}^{\infty} \varphi(u) d u \\
& =-\frac{D}{\tau} \frac{\omega^{2}}{2 \Omega^{2}} \Re[\hat{\varphi}(2 \Omega)]+D \frac{\omega^{2}}{2 \Omega^{2}}
\end{aligned}
$$




$$
\begin{aligned}
B_{4} & =-\frac{D}{\tau} \frac{\omega}{2 \Omega} \int_{0}^{\infty} d u \varphi(u)\left[\sin (2 \Omega u)-\frac{\lambda}{\Omega} \cos (2 \Omega u)\right] \\
& -\frac{D}{\tau} \frac{\lambda}{2} \frac{\omega}{\Omega^{2}} \int_{0}^{\infty} \varphi(u) d u \\
& =\frac{D}{\tau} \frac{\omega}{2 \Omega}\left\{\frac{\lambda}{\Omega} \Re[\hat{\varphi}(2 \Omega)]+\Im[\hat{\varphi}(2 \Omega)]\right\} \\
& -D \frac{\lambda}{2} \frac{\omega}{\Omega^{2}}
\end{aligned}
$$

with $\tau=\Re[\hat{\varphi}(0)]$, and the symbols $\Im[\ldots]$ and $\Re[\ldots]$ stand for the imaginary and the real part of [...], respectively, and the hat over the function $\varphi$ means the Fourier transform of this function, having assumed $\varphi(t)=0$ for $t<0: \hat{\varphi}(\alpha) \equiv \int_{0}^{\infty} \exp [-\mathrm{i} \alpha u] \varphi(u) d u$. The following equalities hold:

$$
A_{4}+B_{3}=D, \quad A_{2}+B_{1}=A_{0}+D .
$$

In the limit of large time scale separation between the ROM and the fast booster, we can use the approximations $\Re[\hat{\varphi}(x \Omega)] \sim \Re[\hat{\varphi}(0)]=\tau$ and $\Im[\hat{\varphi}(x \Omega)] \sim \Im[\hat{\varphi}(0)]=0$ in Equations (A7)-(A13), and we obtain the standard FPE for an oscillator perturbed by a white noise, given in Equation (17).

\section{Appendix C. The Ansatz and the Stationary PDF}

Here, we review Appendix B of [17], to find the reduced stationary PDF for the T variable. We start from the FPE of Equation (17), which, after the shift $h \rightarrow h+\beta D / \omega$, which annuls the mean value of the anomaly thermocline depth, becomes the FPE of Equations (33) and (34). Because we are interested only in the reduced PDF for the $T$ variable, we integrate the $h$ variable in the FPE of Equations (33) and (34), to obtain (we remind again that $D \equiv \epsilon^{2}\left\langle\xi^{2}\right\rangle_{b} \tau$ ):

$$
\partial_{t} p(T ; t)=-\omega \partial_{T}\langle h\rangle_{T}+\left\{\left(\lambda-D \beta^{2}\right) \partial_{T} T+\partial_{T}^{2} D(1+\beta T)^{2}\right\} p(T ; t),
$$

where $p(T ; t) \equiv \int \sigma(h, T ; t) \mathrm{d} h$ is the reduced PDF for the $T$ variable and $\langle h\rangle_{T} \equiv \int h \sigma(h, T ; t) \mathrm{d} h$ is the conditional average value of the $h$ variable (note: $p_{s}(T) \equiv \int \sigma_{s}(h, T) \mathrm{d} h$ ). Because $\omega \sim 0.13$ and $\lambda \sim 0.1$, we are neither in the under-damped, nor in the over-damped standard cases where it is possible to reduce the two-dimensional FPE to the one-dimensional type [42,65,90]. However, we take advantage of the fact that here, we focus our attention on the stationary PDF of the reduced FPE, for which $\partial p(T ; t) / \partial t=0$ in Equation (A16). Thus, observing that the relations among the stationary first and second moments of the $h$ and $T$ variables that we obtain from Equation (23) are the same for the case of a stochastic damped linear oscillator (see also B_16), we make the following ansatz:

$$
\langle h\rangle_{T, s}=\langle h\rangle_{s} p_{s}(T)=0
$$

where the former equality represents the ansatz.

Inserting Equation (A17) in the FPE of Equation (A16), imposing the condition of vanishing current and using the definition $\mu \equiv 1+\lambda /\left(D \beta^{2}\right)$, we obtain:

$$
\beta(2+\mu \beta T) p_{s}(T)+(1+\beta T)^{2} \partial_{T} p_{s}(T)=0 .
$$

From the previous equation, we get two results: the stationary PDF has a maximum (i.e., the $T$ derivative vanishes) for $T=-2 /(\beta \mu)=-2 \beta D /\left(\lambda+D \beta^{2}\right)$; the stationary PDF must vanish for $T=-1 / \beta$ (and for lesser values as well, for obvious physical reasons). As already noted in [17], from Equation (23), for $\left(\lambda-2 \beta^{2} D\right)>0$ (namely, for $\mu>3$ ), the equation of motion of the first and second 
order moments of the ROM converge to a finite value for $t \rightarrow \infty$. It is not difficult to check that the same constraint ensures that also the solution of the FPE of Equation (17) converges, for $t \rightarrow \infty$, to a finite (normalizable) stationary PDF. Thus, under the assumption that $\mu>3$, from Equation (A18), we get the stationary PDF given in Equation (29).

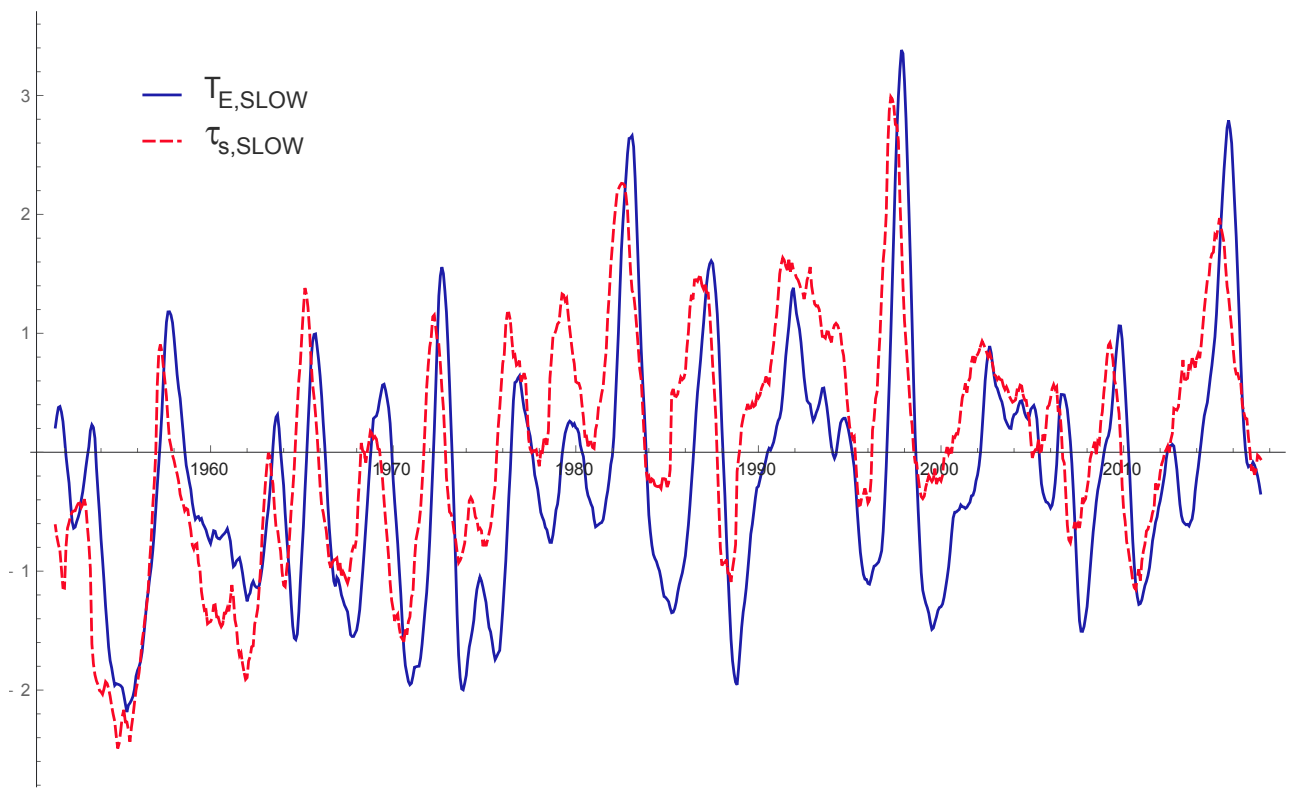

Figure A2. Plot of the 13-month averaged Niño 3 data (blues solid line) and of the 13-month averaged wind stress anomaly (dashed red line). Quantities are normalized by their respective standard deviations. The Niño 3 data are from [50], while the wind stress is proportional to the square of the wind velocity, where the wind data are from [61]. The wind stress has been averaged on the equatorial strip defined by $\left(5^{\circ} \mathrm{S}-5^{\circ} \mathrm{N}\right) \times\left(120^{\circ} \mathrm{W}-180^{\circ} \mathrm{W}\right)$

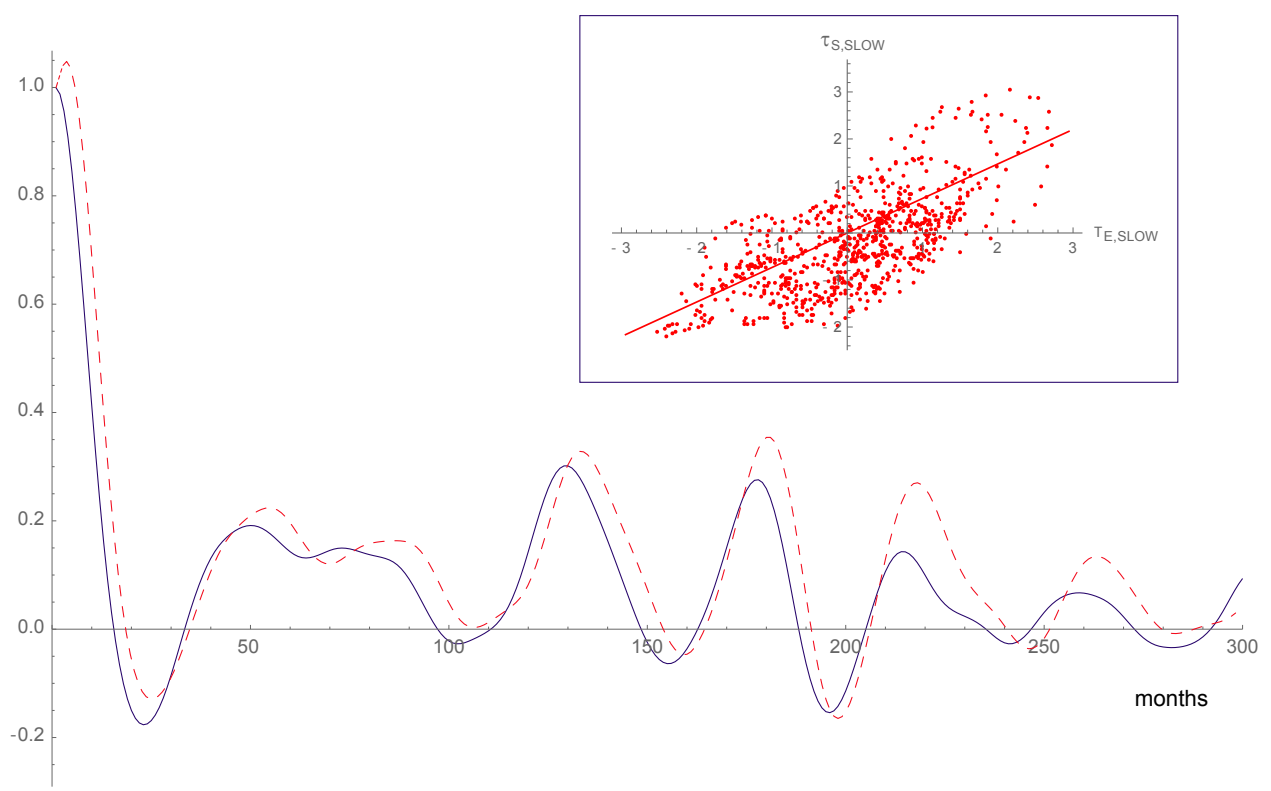

Figure A3. Blue solid line: normalized auto-correlation of the one-year averaged Niño 3 index: $\left\langle T_{E, \text { slow }}(0) T_{E, \text { slow }}(t)\right\rangle_{s} /\left\langle T_{E \text {, slow }}(0) T_{E \text {,slow }}(0)\right\rangle_{s}$. Red-dashed line: cross-correlation between the $\tau_{s, \text { slow }}$ and $T_{E \text {,slow }}$, normalized with the initial value: $\left\langle\tau_{s, s l o w}(0) T_{E, \text { slow }}(t)\right\rangle_{s} /\left\langle\tau_{s, \text { slow }}(0) T_{E, \text { slow }}(0)\right\rangle_{s}$. We can see that they are very similar to each other. Insert: $\tau_{s, s l o w}$ vs. $T_{E, \text { slow }}$ (red dots). Data from NOAA [50](Niño 3) and [61] (wind). 


\section{References}

1. Larkin, N.K.; Harrison, D.E. ENSO Warm (El Niño) and Cold (La Niña) Event Life Cycles: Ocean Surface Anomaly Patterns, Their Symmetries, Asymmetries, and Implications. J. Clim. 2002, 15, 1118-1140. [CrossRef]

2. Kug, J.S.; Ham, Y.G. Are there two types of La Nina? Geophys. Res. Lett. 2011, 38. [CrossRef]

3. An, S.I. Interdecadal changes in the El Nino-La Nina asymmetry. Geophys. Res. Lett. 2004, 31. [CrossRef]

4. An, S.I.; Kim, J.W. Role of nonlinear ocean dynamic response to wind on the asymmetrical transition of El Niño and La Niña. Geophys. Res. Lett. 2017, 44, 393-400. [CrossRef]

5. Dommenget, D.; Bayr, T.; Frauen, C. Analysis of the non-linearity in the pattern and time evolution of El Niño southern oscillation. Clim. Dyn. 2013, 40, 2825-2847. [CrossRef]

6. Frauen, C.; Dommenget, D. El Niño and La Niña amplitude asymmetry caused by atmospheric feedbacks. Geophys. Res. Lett. 2010, 37, L18801. [CrossRef]

7. Moore, A.M.; Kleeman, R. The dynamics of error growth and predictability in a coupled model of ENSO. Q. J. R. Meteorol. Soc. 1996, 122, 1405-1446. [CrossRef]

8. Penland, C.; Sardeshmukh, P.D. The Optimal Growth of Tropical Sea Surface Temperature Anomalies. J. Clim. 1995, 8, 1999-2024. [CrossRef]

9. Weiss, J.B. Fluctuation properties of steady-state Langevin systems. Phys. Rev. E 2007, 76, 061128. [CrossRef] [PubMed]

10. Jin, F.F. An Equatorial Ocean Recharge Paradigm for ENSO. Part II: A Stripped-Down Coupled Model. J. Atmos. Sci. 1997, 54, 830-847. [CrossRef]

11. Jin, F.F. An Equatorial Ocean Recharge Paradigm for ENSO. Part I: Conceptual Model. J. Atmos. Sci. 1997, 54, 811-829. [CrossRef]

12. Burgers, G.; Jin, F.F.; van Oldenborgh, G.J. The simplest ENSO recharge oscillator. Geophys. Res. Lett. 2005, 32, L13706. [CrossRef]

13. Capotondi, A.; Wittenberg, A.; Masina, S. Spatial and temporal structure of Tropical Pacific interannual variability in 20th century coupled simulations. Ocean Model. 2006, 15, 274-298. [CrossRef]

14. Jin, F.F.; Lin, L.; Timmermann, A.; Zhao, J. Ensemble-mean dynamics of the ENSO recharge oscillator under state-dependent stochastic forcing. Geophys. Res. Lett. 2007, 34, L03807. [CrossRef]

15. Levine, A.F.Z.; Jin, F.F. Noise-Induced Instability in the ENSO Recharge Oscillator. J. Atmos. Sci. 2010, 67, 529-542. [CrossRef]

16. Ren, H.L.; Jin, F.F. Recharge Oscillator Mechanisms in Two Types of ENSO. J. Clim. 2013, 26, 6506-6523. [CrossRef]

17. Bianucci, M. Analytical probability density function for the statistics of the ENSO phenomenon: Asymmetry and power law tail. Geophys. Res. Lett. 2016, 43, 386-394. [CrossRef]

18. Battisti, D.S.; Hirst, A.C. Interannual variability in a tropical atmosphere-ocean model: Influence of the basic state, ocean geometry and nonlinearity. J. Atmos. Sci. 1989, 46, 1687-1712. [CrossRef]

19. Mantua, N.J.; Battisti, D.S. Evidence for the Delayed Oscillator Mechanism for ENSO: The "Observed" Oceanic Kelvin Mode in the Far Western Pacific. J. Phys. Oceanogr. 1994, 24, 691-699. [CrossRef]

20. Im, S.H.; An, S.I.; Kim, S.T.; Jin, F.F. Feedback processes responsible for El Niño-La Niña amplitude asymmetry. Geophys. Res. Lett. 2015, 42, 5556-5563. [CrossRef]

21. Sardeshmukh, P.D.; Penland, C. Understanding the distinctively skewed and heavy tailed character of atmospheric and oceanic probability distributions. Chaos 2015, 25, 036410. [CrossRef] [PubMed]

22. Sardeshmukh, P.D.; Sura, P. Reconciling Non-Gaussian Climate Statistics with Linear Dynamics. J. Clim. 2009, 22, 1193-1207. [CrossRef]

23. Sura, P.; Sardeshmukh, P.D. A global view of air-sea thermal coupling and related non-Gaussian \{SST\} variability. Atmos. Res. 2009, 94, 140-149. [CrossRef]

24. Sardeshmukh, P.D.; Compo, G.P.; Penland, C. Need for Caution in Interpreting Extreme Weather Statistics. J. Clim. 2015, 28, 9166-9187. [CrossRef]

25. Penland, C.; Sardeshmukh, P.D. Alternative interpretations of power-law distributions found in nature. Chaos Interdiscip. J. Nonlinear Sci. 2012, 22, 023119. [CrossRef] [PubMed] 
26. Bianucci, M.; Merlino, S. Non Standard Fluctuation Dissipation Processes in Ocean-Atmosphere Interaction and for General Hamiltonian or Non Hamiltonian Phenomena: Analytical Results; Mathematics Research Developments, Nova Science Publisher: Hauppauge, NY, USA, 2017.

27. Bianucci, M. On the correspondence between a large class of dynamical systems and stochastic processes described by the generalized Fokker Planck equation with state-dependent diffusion and drift coefficients. J. Stat. Mech. Theory Exp. 2015, 2015, P05016. [CrossRef]

28. Dorfman, J.R. An Introduction to Chaos in Nonequilibrium Statistical Mechanics; Cambridge Lecture Notes in Physics, Cambridge University Press: Cambridge, UK, 1999.

29. Zwanzig, R., Ed. Nonequilibrium Statistical Mechanics; Oxford University Press: Oxford, UK, 2001.

30. Chorin, A.J.; Hald, O.H.; Kupferman, R. Optimal prediction and the Mori-Zwanzig representation of irreversible processes. Proc. Natl. Acad. Sci. USA 2000, 97, 2968-2973. [CrossRef] [PubMed]

31. Grigolini, P.; Marchesoni, F. Basic Description of the Rules Leading to the Adiabatic Elimination of Fast Variables. In Advances in Chemical Physics: Memory Function Approaches to Stochastich Problems in Condensed Matter; Evans, M.W., Grigolini, P., Parravicini, G.P., Eds.; John Wiley \& Sons: New York, NY, USA, 1985; Volume 62, p. 556.

32. Bianucci, M. Using some results about the Lie evolution of differential operators to obtain the Fokker-Planck equation for non-Hamiltonian dynamical systems of interest. J. Math. Phys. 2018, 59, 053303. [CrossRef]

33. Bianucci, M. Large Scale Emerging Properties from Non Hamiltonian Complex Systems. Entropy 2017, 19, 302. [CrossRef]

34. Smoluchowski, M.V. Irregularity in the distribution of gaseous molecules and its influence. Boltzmann Festschr. 1904, 626-641.

35. Smoluchowski, M.V. Theory of the Brownian movements. Bull. Acad. Sci. Crac. 1906, 577-602.

36. Schadschneider, A.; Chowdhury, D.; Nishinari, K. (Eds.) Stochastic Transport in Complex Systems. From Molecules to Vehicles; Elsevier: Amsterdam, The Netherlands, 2010.

37. Kampen, N.V. (Ed.) Stochastic Processes in Physics and Chemistry, 3rd ed.; Elsevier: Amsterdam, The Netherlands, 2007.

38. Risken, H. The Fokker-Planck Equation. Methods of Solution and Applications; Springer: Berlin/Heidelberg, Germany, 1996; Volume 18,

39. Kubo, R.; Toda, M.; Hashitsume, N. Statistical Physics II. Nonequilibrium Statistical Mechanics; Springer: Berlin/Heidelberg, Germany, 1985; Volume 31,

40. Ding, M.; Rangarajan, G. First Passage Time Problem: A Fokker-Planck Approach. In New Directions in Statistical Physics: Econophysics, Bioinformatics, and Pattern Recognition; Wille, L.T., Ed.; Springer: Berlin/Heidelberg, Germany, 2004; pp. 31-46.

41. Kramers, H. Brownian motion in a field of force and the diffusion model of chemical reactions. Physica 1940, 7, 284-304. [CrossRef]

42. Bianucci, M.; Grigolini, P.; Palleschi, V. Beyond the linear approximations of the conventional approaches to the theory of chemical relaxation. J. Chem. Phys. 1990, 92, 3427-3441. [CrossRef]

43. Bianucci, M. Ordinary chemical reaction process induced by a unidimensional map. Phys. Rev. E 2004, 70, 026107. [CrossRef] [PubMed]

44. Revelli, J.A.; Budde, C.E.; Wio, H.S. Diffusion in fluctuating media: First passage time problem. Phys. Lett. A 2002, 306, 104-109. [CrossRef]

45. Kulkarni, V.G.; Tzenova, E. Mean first passage times in fluid queues. Oper. Res. Lett. 2002, 30, $308-318$. [CrossRef]

46. Giorno, V.; Nobile, A.; Ricciardi, L. Single neuron's activity: On certain problems of modeling and interpretation. Biosystems 1997, 40, 65-74. [CrossRef]

47. Tuckwell, H.C. (Ed.) Introduction to Theoretical Neurobiology; Cambridge Studies in Mathematical Biology, Cambridge University Press: Cambridge, UK, 2006.

48. Kurzyński, M.; Chełminiak, P. Mean First-Passage Time in the Stochastic Theory of Biochemical Processes. Application to Actomyosin Molecular Motor. J. Stat. Phys. 2003, 110, 137-181. [CrossRef]

49. Brics, M.; Kaupuzs, J.; Mahnke, R. How to solve Fokker-Planck equation treating mixed eigenvalue spectrum? Condens. Matter Phys. 2013, 16, 1-13. [CrossRef]

50. NOAA Climate Prediction Center. Available online: https://www.cpc.ncep.noaa.gov/data/indices/ (accessed on 20 June 2018). 
51. Philander, S.G.H. The Response of Equatorial Oceans to a Relaxation of the Trade Winds. J. Phys. Oceanogr. 1981, 11, 176-189. [CrossRef]

52. Jin, F.F.; Neelin, J.D. Modes of Interannual Tropical Ocean-Atmosphere Interaction-A Unified View. Part I: Numerical Results. J. Atmos. Sci. 1993, 50, 3477-3503. [CrossRef]

53. Neelin, J.D.; Jin, F.F. Modes of Interannual Tropical Ocean-Atmosphere Interaction-A Unified View. Part II: Analytical Results in the Weak-Coupling Limit. J. Atmos. Sci. 1993, 50, 3504-3522. [CrossRef]

54. Khas'minskii, R. A Limit Theorem for the Solutions of Differential Equations with Random Right-Hand Sides. Theory Probab. Appl. 1966, 11, 390-406. [CrossRef]

55. Gallavotti, G.; Cohen, E.G.D. Dynamical ensembles in stationary states. J. Stat. Phys. 1995, 80, 931-970. [CrossRef]

56. Gallavotti, G.; Cohen, E.G.D. Dynamical Ensembles in Nonequilibrium Statistical Mechanics. Phys. Rev. Lett. 1995, 74, 2694-2697. [CrossRef] [PubMed]

57. Terwiel, R. Projection operator method applied to stochastic linear differential equations. Physica 1974, 74, 248-265. [CrossRef]

58. Da-jin, W.; Li, C.; Bo, Y. Probability Evolution and Mean First-Passage Time for Multidimensional Non-Markovian Processes. Commun. Theor. Phys. 1989, 11, 379. [CrossRef]

59. Kampen, N.V. Elimination of fast variables. Phys. Rep. 1985, 124, 69-160. [CrossRef]

60. Kim, S.T.; Jin, F.F. An ENSO stability analysis. Part II: Results from the twentieth and twenty-first century simulations of the CMIP3 models. Clim. Dyn. 2011, 36, 1609-1627. [CrossRef]

61. NOAA ESRL, NCEP/NCAR Reanalysis Data. Available online: https://www.esrl.noaa.gov/psd/data/ gridded/data.ncep.reanalysis.derived.surfaceflux.html (accessed on 20 June 2018).

62. Wen, C.; Kumar, A.; Xue, Y.; McPhaden, M.J. Changes in Tropical Pacific Thermocline Depth and Their Relationship to ENSO after 1999. J. Clim. 2014, 27, 7230-7249. [CrossRef]

63. Bianucci, M. Nonconventional fluctuation dissipation process in non-Hamiltonian dynamical systems. Int. J. Mod. Phys. B 2015, 30, 1541004. [CrossRef]

64. Grigolini, P. The projection approach to the Fokker-Planck equation: Applications to phenomenological stochastic equations with coloured noises. In Noise in Nonlinear Dynamical Systems; Moss, F., McClintock, P.V.E., Eds.; Cambridge University Press: Cambridge, UK, 1989; Volume 1, Chapter 5, p. 161.

65. Bianucci, M.; Grigolini, P. Nonlinear and non Markovian fluctuation-dissipation processes: A Fokker-Planck treatment. J. Chem. Phys. 1992, 96, 6138-6148. [CrossRef]

66. Bianucci, M.; Mannella, R.; West, B.J.; Grigolini, P. From dynamics to thermodynamics: Linear response and statistical mechanics. Phys. Rev. E 1995, 51, 3002-3022. [CrossRef]

67. Hayashi, M.; Jin, F.F. Subsurface Nonlinear Dynamical Heating and ENSO Asymmetry. Geophys. Res. Lett. 2017, 44. [CrossRef]

68. Kapur, A.; Zhang, C. Multiplicative MJO Forcing of ENSO. J. Clim. 2012, 25, 8132-8147. [CrossRef]

69. Capotondi, A.; Sardeshmukh, P.D.; Ricciardulli, L. The Nature of the Stochastic Wind Forcing of ENSO. J. Clim. 2018, 31, 8081-8099. [CrossRef]

70. Sura, P. On non-Gaussian SST variability in the Gulf Stream and other strong currents. Ocean Dyn. 2010, 60,155-170. [CrossRef]

71. Sura, P. A general perspective of extreme events in weather and climate. Atmos. Res. 2011, 101, 1-21. [CrossRef]

72. Frankignoul, C. Sea surface temperature anomalies, planetary waves, and air-sea feedback in the middle latitudes. Rev. Geophys. 1985, 23, 357-390. [CrossRef]

73. Papanicolaou, G.C.; Kohler, W. Asymptotic theory of mixing stochastic ordinary differential equations. Commun. Pure Appl. Math. 1974, 27, 641-668. [CrossRef]

74. Gardiner, C. Stochastic Methods. A Handbook for the Natural and Social Sciences, 4 ed.; Springer: Berlin/Heidelberg, Germany, 2009; Volume 13, pp. 18, 447.

75. Prasad, P.; Ravindran, R. Partial Differential Equations; Wiley Eastern, Delhi and John Wiley \& Sons, New York, NY, USA, 1985.

76. IRI/LDEO Climate Data Library. Available online: http://iridl.ldeo.columbia.edu/ (accessed on 20 June 2018). 
77. Bianucci, M.; Capotondi, A.; Merlino, S.; Mannella, R. Estimate of the average timing for strong El Niño events using the recharge oscillator model with a multiplicative perturbation. Chaos Interdiscip. J. Nonlinear Sci. 2018, 28, 103118. [CrossRef] [PubMed]

78. Tokyo Climate Center. Available online: http:/ / ds.data.jma.go.jp/tcc/tcc/ (accessed on 20 January 2018).

79. Gardiner, C. Stochastic Methods; Springer: Berlin/Heidelberg, Germany, 2009; Volume 20.

80. Wang, M.C.; Uhlenbeck, G.E. On the Theory of the Brownian Motion II. Rev. Mod. Phys. 1945, 17, $323-342$. [CrossRef]

81. Siegert, A.J.F. On the First Passage Time probability problem. Phys. Rev. 1951, 81, 617-623. [CrossRef]

82. KAMPEN, N.V. Chapter $\{\mathrm{VIII}\}-\{\mathrm{THE}\}$ FOKKER-PLANCK \{EQUATION\}. In Stochastic Processes in Physics and Chemistry, 3rd ed.; KAMPEN, N.V., Ed.; North-Holland Personal Library, Elsevier: Amsterdam, The Netherlands, 2007; pp. 193-218.

83. McGregor, S.; Timmermann, A.; Timm, O. A unified proxy for ENSO and PDO variability since 1650. Clim. Past 2010, 6, 1-17. [CrossRef]

84. Bianucci, M.; Mannella, R.; Fan, X.; Grigolini, P.; West, B.J. Standard fluctuation-dissipation process from a deterministic mapping. Phys. Rev. E 1993, 47, 1510-1519. [CrossRef]

85. Zhang, C.; Gottschalck, J. SST Anomalies of ENSO and the Madden-Julian Oscillation in the Equatorial Pacific. J. Clim. 2002, 15, 2429-2445. [CrossRef]

86. Li, R.C.Y.; Zhou, W.; Chan, J.C.L.; Huang, P. Asymmetric Modulation of Western North Pacific Cyclogenesis by the Madden-Julian Oscillation under ENSO Conditions. J. Clim. 2012, 25, 5374-5385. [CrossRef]

87. Zavala-Garay, J.; Zhang, C.; Moore, A.M.; Kleeman, R. The Linear Response of ENSO to the Madden-Julian Oscillation. J. Clim. 2005, 18, 2441-2459. [CrossRef]

88. Hendon, H.H.; Wheeler, M.C.; Zhang, C. Seasonal Dependence of the MJO-ENSO Relationship. J. Clim. 2007, 20, 531-543. [CrossRef]

89. Dijkstra, H.A.; Burgers, G. Fluid dynamics of el Niño variability. Annu. Rev. Fluid Mech. 2002, 34, 531-558. [CrossRef]

90. Carmeli, B.; Nitzan, A. Non-Markovian theory of activated rate processes. I. Formalism. J. Chem. Phys. 1983, 79, 393-404. [CrossRef] 